\title{
PARTIAL REGULARITY OF MINIMIZERS OF HIGHER ORDER INTEGRALS WITH $(p, q)$-GROWTH
}

\section{SABINE SCHEMM ${ }^{1}$}

Abstract. We consider higher order functionals of the form

$$
F[u]=\int_{\Omega} f\left(D^{m} u\right) \mathrm{d} x \quad \text { for } u: \mathbb{R}^{n} \supset \Omega \rightarrow \mathbb{R}^{N},
$$

where the integrand $f: \bigodot^{m}\left(\mathbb{R}^{n}, \mathbb{R}^{N}\right) \rightarrow \mathbb{R}, m \geq 1$ is strictly quasiconvex and satisfies a non-standard growth condition. More precisely we assume that $f$ fulfills the $(p, q)$-growth condition

$$
\gamma|A|^{p} \leq f(A) \leq L\left(1+|A|^{q}\right) \quad \text { for all } A \in \bigodot^{m}\left(\mathbb{R}^{n}, \mathbb{R}^{N}\right),
$$

with $\gamma, L>0$ and $1<p \leq q<\min \left\{p+\frac{1}{n}, \frac{2 n-1}{2 n-2} p\right\}$. We study minimizers of the functional $F[\cdot]$ and prove a partial $C_{\text {loc }}^{m, \alpha}$-regularity result.

Mathematics Subject Classification. 49N60, 49N99, 49J45.

Received May 2nd, 2008.

Published online April 23, 2010.

\section{INTRODUCTION}

In this paper we are interested in the interior regularity theory for minimizers of higher order functionals with $(p, q)$-growth. For $m \geq 1$ we consider variational integrals of the type

$$
F[u]=\int_{\Omega} f\left(D^{m} u\right) \mathrm{d} x,
$$

where $\Omega$ is a bounded domain in $\mathbb{R}^{n}, u \in W^{m, 1}\left(\Omega, \mathbb{R}^{N}\right), n \geq 2, N \geq 1$ and $f \in C^{2}\left(\bigodot^{m}\left(\mathbb{R}^{n}, \mathbb{R}^{N}\right)\right)($ here $\bigodot^{m}\left(\mathbb{R}^{n}, \mathbb{R}^{N}\right)$ denotes the vectorspace of symmetric $m$-linear functions on $\mathbb{R}^{n}$ with values in $\left.\mathbb{R}^{N}\right)$. Furthermore, we assume that the integrand $f$ is a strictly $W^{m, p}$-quasiconvex function (see (H3s), Sect. 1 for the definition) and satisfies the $(p, q)$-growth condition

$$
\gamma|A|^{p} \leq f(A) \leq L\left(1+|A|^{q}\right)
$$

Keywords and phrases. Higher order functionals, non-standard growth, regularity theory.

1 Mathematisches Institut der Friedrich-Alexander-Universität Erlangen-Nürnberg, Bismarckstraße 1 1/2, 91054 Erlangen, Germany. schemm@mi.uni-erlangen.de 
for every $A \in \bigodot^{m}\left(\mathbb{R}^{n}, \mathbb{R}^{N}\right)$ with $\gamma, L>0$ and $1<p \leq q<\min \left\{p+\frac{1}{n}, \frac{2 n-1}{2 n-2} p\right\}$. In the following we show existence and partial $C_{\mathrm{loc}}^{m, \alpha}$ regularity of $W^{m, p}$-minimizers of the functional (0.1) with respect to some fixed boundary values. Thereby $W^{m, p}$-minimizers of $F[\cdot]$ are defined as follows:

Definition 0.1. Let $p \in[1, \infty]$. A map $u \in W^{m, p}(\Omega, \mathbb{R})$ with $F[u]<\infty$ is called a $W^{m, p}$-minimizer of $F[\cdot]$ if

$$
F[u] \leq F[u+\varphi] \quad \text { for every } \varphi \in W_{0}^{m, p}\left(\Omega, \mathbb{R}^{N}\right)
$$

For second order functionals, i.e. $m=1$, there are numerous papers dealing with existence and partial regularity of minimizers of quasiconvex functionals (see (1.7), Sect. 1 for the definition) which satisfy the standard $p$-growth condition

$$
\gamma|A|^{p} \leq f(A) \leq L\left(1+|A|^{p}\right)
$$

for every $A \in \bigodot^{m}\left(\mathbb{R}^{n}, \mathbb{R}^{N}\right)$ and $1<p \leq \infty$. Since under certain circumstances quasiconvexity is a necessary and sufficient condition for sequential weak lower semicontinuity of $F[\cdot]$ it turned out to be a primary hypothesis in the existence theory of minimizers (see Sect. 1.1 for further details). Strengthening this condition slightly, Evans derived a first regularity result in [20]. More precisely, he showed for the superquadratic case $(p \geq 2)$ that minimizers of strictly quasiconvex functionals are partially $C_{\mathrm{loc}}^{1, \alpha}$-regular. This result was extended to the subquadratic case $(1<p<2)$ in [11]. Further papers concerning this topic are for example $[2,5,13,15,16,23,26]$ and also [31] investigating the Hausdorff-dimension of the singular set. For higher order functionals partial regularity results have been shown in $[27,32]$ (see also [45]).

In the last years functionals with non-standard growth conditions attracted more attention. In this paper, we focus on the so called $(p, q)$-growth condition $(0.2)$ which was introduced by Marcellini. Examples in the late 80 's (see $[25,29,35]$ ) show that we cannot expect regularity in general if $q$ is large enough with respect to $p$. As a start Marcellini studied in the scalar case variational functionals and elliptic equations with $(p, q)$-growth (see $[36,37]$ ) and deduced basic regularity results. Also various contributions in the vectorial case appeared, see for first examples [4,38,41]. In particular, in [41] it is proved under the assumptions that $f$ is strictly convex and satisfies the $(p, q)$-growth condition with $2 \leq p<q<\min \left\{p+1, \frac{p n}{n-1}\right\}$ that minimizers of $F[\cdot]$ are partially $C_{\text {loc }}^{1, \alpha}$-regular. Imposing an additional growth condition on the second derivatives of $f$ in [7] this result was gained under the weaker condition $q<p \frac{n+2}{n}$ and for integrands with $x$ dependency in [8] (see also [12]). For a higher order version of [41] we refer to [10].

Furthermore, results concerning higher integrability of gradients of minimizers have been established in [18,19].

A first regularity result for quasiconvex functionals with $m=1$ was recently given in [43]. There it is proved that if $f$ is strictly $W^{1, p}$-quasiconvex (see (H2), Sect. 1 for the definition) and satisfies the $(p, q)$-growth condition (0.2) with

$$
1<p \leq q<p+\frac{\min \{2, p\}}{2 n},
$$

then $u$ is partial $C_{\text {loc }}^{1, \alpha}$-regular. The same author also follows an alternative approach of treating regularity of minimizers [44]. This approach relies on a natural relaxation procedure in the spirit of the Lebesgue-Serrin extension which was introduced in $[9,21,34]$.

Following the strategy in [43] this paper aims to give a higher order version of the result in [43]. Under the condition

$$
1<p \leq q<\min \left\{p+\frac{1}{n}, \frac{2 n-1}{2 n-2} p\right\}
$$

we show that a minimizer $u$ of $(0.1)$ is partial $C_{\text {loc }}^{m, \alpha}$-regular. We remark that condition $(0.4)$ is more general than (0.3). But this improvement has only technical reasons. It appears also in [42] where strong local minimizers of first order functionals are treated. We show the partial regularity of $u$ via $\mathcal{A}$-harmonic approximation, a method developed in [14]. The main step to proof the regularity result is the derivation of a decay estimate 
for the excess function

$$
\Phi(\rho)=\left(f_{B_{\rho}\left(x_{0}\right)}\left|V\left(D^{m} u\right)-V\left(\left(D^{m} u\right)_{x_{0}, \rho}\right)\right|^{2} \mathrm{~d} x\right)^{\frac{1}{2}},
$$

where $\left(D^{m} u\right)_{x_{0}, \rho}:=f_{B_{\rho}\left(x_{0}\right)} D^{m} u \mathrm{~d} x$. For the proof of this decay estimate we need as a key tool the trace preserving operator $T$ which was introduced by Fonseca and Malý in [21] and extended by the same authors to higher orders in [22].

\section{Preliminaries and Statement of the Result}

In this section we state our main result concerning partial regularity of minimizers. Let $\Omega \subset \mathbb{R}^{n}$ be a bounded domain, $1<p \leq q<\infty$ and $u \in W^{m, 1}\left(\Omega, \mathbb{R}^{N}\right), n \geq 2, N \geq 1$. We consider $W^{m, p}$-minimizers of the functional

$$
F[u]=\int_{\Omega} f\left(D^{m} u\right) \mathrm{d} x,
$$

where $f$ is a $C^{2}$-function which satisfies some of the following conditions:

(H1) q-Growth: There exists a bound $L>0$ such that we have

$$
0 \leq f(A) \leq L\left(1+|A|^{q}\right) \quad \text { for every } A \in \bigodot^{m}\left(\mathbb{R}^{n}, \mathbb{R}^{N}\right) .
$$

(H2) $p$-Coercivity: There is a coercivity constant $\gamma>0$ such that holds

$$
f(A) \geq \gamma|A|^{p} \quad \text { for every } A \in \bigodot^{m}\left(\mathbb{R}^{n}, \mathbb{R}^{N}\right) .
$$

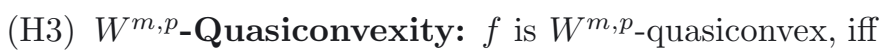

$$
\int_{\Omega} f\left(A+D^{m} \varphi\right) \mathrm{d} x \geq \int_{\Omega} f(A) \mathrm{d} x
$$

for all $A \in \bigodot^{m}\left(\mathbb{R}^{n}, \mathbb{R}^{N}\right)$ and for all $\varphi \in W_{0}^{m, p}\left(\Omega, \mathbb{R}^{N}\right)$.

Remark. The $W^{m, p}$-quasiconvexity condition has been introduced by Ball and Murat in [6]. There it is proved for $m=1$ that (H3) is a necessary condition for the sequential weak lower semicontinuity of the functional $F[\cdot]$ on $W^{m, p}\left(\Omega, \mathbb{R}^{N}\right.$ ) (an easy adaptation of the arguments gives this result also for $m>1$ ). We will later see that the $W^{m, p}$-quasiconvexity condition together with the $q$-growth condition (H1) and the $p$-coercivity condition (H2) implies the existence of at least one minimizer.

(H3s) Strict $W^{m, p}$-quasiconvexity: $f$ is strictly non-degenerate $W^{m, p}$-quasiconvex, i.e. for each $M>0$ there is a convexity constant $\lambda_{M}>0$ such that

$$
\int_{\Omega}\left(f\left(A+D^{m} \varphi\right)-f(A)\right) \mathrm{d} x \geq \lambda_{M} \int_{\Omega}\left(1+\left|D^{m} \varphi\right|^{2}\right)^{\frac{p-2}{2}}\left|D^{m} \varphi\right|^{2} \mathrm{~d} x
$$

for all $A \in \bigodot^{m}\left(\mathbb{R}^{n}, \mathbb{R}^{N}\right)$ with $|A| \leq M+1$ and for all $\varphi \in W_{0}^{m, p}\left(\Omega, \mathbb{R}^{N}\right)$.

Remark. This strong quasiconvexity assumption is necessary in particular for the proof of the partial regularity result, whereas for the existence result, condition (H3) is sufficient. Note that (H3s) is even stronger than the strict quasiconvexity of $f$ that has to be assumed in the corresponding standard growth case: There, we require that (1.1) be satisfied for all smooth functions $\varphi \in C_{0}^{\infty}\left(\Omega, \mathbb{R}^{N}\right)$. 
Due to the $q$-growth of the functional, this amounts to allowing all $\varphi \in W^{m, q}\left(\Omega, \mathbb{R}^{N}\right)$ as test functions. This condition does not suffice here, since we want to test with functions which are in general only in $W^{m, p}\left(\Omega, \mathbb{R}^{N}\right)$ and not in $W^{m, q}\left(\Omega, \mathbb{R}^{N}\right)$. Therefore, we assume that $f$ is $W^{m, p}$-quasiconvex.

Remark 1.1. The following properties of $f$ are consequences of the preceding assumptions.

(1) From the quasiconvexity condition (H3s) and the growth condition (H1) we can derive (see e.g. [33]) that

$$
|D f(A)| \leq c(1+|A|)^{q-1} \quad \text { for every } A \in \bigodot^{m}\left(\mathbb{R}^{n}, \mathbb{R}^{N}\right),
$$

with $c=c(n, N, q, L)$.

(2) Let $M>0$ and consider $A \in \bigodot^{m}\left(\mathbb{R}^{n}, \mathbb{R}^{N}\right)$ with $|A| \leq M+1$. Then (H3s) implies that $D^{2} f(A)$ fulfills the strong Legendre-Hadamard condition, i.e. we have

$$
D^{2} f(A)\left(\xi^{m} \otimes \eta, \xi^{m} \otimes \eta\right) \geq \frac{2 \lambda_{M}}{m !}|\xi|^{2 m}|\eta|^{2}
$$

for all $\xi \in \mathbb{R}^{n}$ and $\eta \in \mathbb{R}^{N}$, see [32] and [39], Theorem 7 .

(3) We have no explicit growth condition on the second derivatives of $f$. But here it is sufficient that for every $M>0$ there exists a constant $K_{M}<\infty$ such that there holds

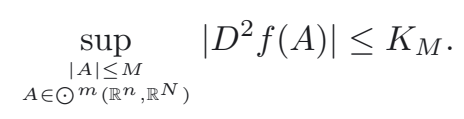

(4) Since $f$ is a $C^{2}$ function for each $M>0$ exists a monotone non-decreasing, concave function $\nu_{M}$ : $[0, \infty) \rightarrow[0, \infty)$ continuous at 0 such that $\nu_{M}(0)=0$ and

$$
\left|D^{2} f(A)-D^{2} f(B)\right| \leq \nu_{M}(|A-B|)
$$

for any $A, B \in \bigodot^{m}\left(\mathbb{R}^{n}, \mathbb{R}^{N}\right)$ with $|A| \leq M+1$ and $|B| \leq M+1$.

We can now formulate our main result:

Theorem 1.2. Let

$$
1<p \leq q<\min \left\{p+\frac{1}{n}, \frac{2 n-1}{2 n-2} p\right\}
$$

and suppose that $f \in C^{2}$ satisfies (H1) and (H3s). Then for each $W^{m, p}\left(\Omega, \mathbb{R}^{N}\right)$-minimizer (see Def. 0.1) of the functional $F[u]=\int_{\Omega} f\left(D^{m} u\right) \mathrm{d} x$ there exists an open subset $\Omega_{0} \subset \Omega$ with

$$
\mathcal{L}^{n}\left(\Omega \backslash \Omega_{0}\right)=0 \quad \text { and } \quad u \in C_{\mathrm{loc}}^{m, \alpha}\left(\Omega_{0}, \mathbb{R}^{N}\right)
$$

for every $0<\alpha<1$. Furthermore $\Omega \backslash \Omega_{0}=\Sigma_{1} \cup \Sigma_{2}$ where

$$
\begin{aligned}
& \Sigma_{1}=\left\{x_{0} \in \Omega: \liminf _{\rho \backslash 0} \underset{B_{\rho}\left(x_{0}\right)}{f}\left|D^{m} u-\left(D^{m} u\right)_{x_{0}, \rho}\right|^{p} \mathrm{~d} x>0\right\} \\
& \Sigma_{2}=\left\{x_{0} \in \Omega: \limsup _{\rho \backslash 0}\left|\left(D^{m} u\right)_{x_{0}, \rho}\right|=\infty\right\}
\end{aligned}
$$

\subsection{Existence}

Although our main result concerns the regularity of minimizers for the sake of completeness we also want to investigate whether minimizers of $F$ exists. 
First Morrey [40] has proved for $m=1$ that the quasiconvexity condition on $f$ is a necessary and sufficient condition for weak lower semicontinuity of certain functionals on certain Sobolev spaces. Thereby $f$ is called quasiconvex if it fulfills

$$
\int_{\Omega} f(A) \mathrm{d} x \leq \int_{\Omega} f\left(A+D^{m} \varphi\right) \mathrm{d} x
$$

for all $\varphi \in C_{0}^{\infty}\left(\Omega, \mathbb{R}^{N}\right)$ and all $A \in \bigodot^{m}\left(\mathbb{R}^{n}, \mathbb{R}^{N}\right)$. Later Acerbi and Fusco [1] showed for $m=1$ that if $f$ is a continuous function and satisfies the $p$-growth condition

$$
0 \leq f(A) \leq L\left(1+|A|^{p}\right) \quad\left(L>0,1 \leq p<\infty, A \in \bigodot^{m}\left(\mathbb{R}^{n}, \mathbb{R}^{N}\right)\right)
$$

then the related functional $F[u]=\int_{\Omega} f\left(D^{m} u\right) \mathrm{d} x$ is sequentially weakly lower semicontinuous on $W^{m, p}\left(\Omega, \mathbb{R}^{N}\right)$ iff $f$ is quasiconvex. For a higher order version of this result we refer to Meyers [39] and Guidorzi and Poggiolini [28]. Assuming additionally the following coercivity condition

$$
\frac{1}{L}\left(1+|A|^{p}\right) \leq f(A) \quad \text { for all } A \in \bigodot^{m}\left(\mathbb{R}^{n}, \mathbb{R}^{N}\right)
$$

it is known - by the direct method in the calculus of variations - that for given boundary data there exists at least one minimizer $u \in W^{m, p}\left(\Omega, \mathbb{R}^{N}\right)$ of the functional $F$.

Now, if we have $(p, q)$-growth in $[21,30]$ it is shown for $m=1$ and $1<p \leq q<\frac{n p}{n-1}$ that the quasiconvexity condition (1.7) together with the $q$-growth condition $(H 1)$ implies that $F[\cdot]$ is lower semicontinuous with respect to weak $W^{1, p}$-convergence of $W^{1, q}$-functions. Then, using the $p$-coercivity condition $(H 2)$ we only get that for given boundary data there exists a $u \in W^{1, p}\left(\Omega, \mathbb{R}^{N}\right)$ such that

$$
F[u] \leq F[v] \quad \text { for all } v \in W_{\mathrm{loc}}^{1, q}\left(\Omega, \mathbb{R}^{N}\right) \cap\left[u+W_{0}^{1, p}\left(\Omega, \mathbb{R}^{N}\right)\right]
$$

But if we use instead of the quasiconvexity condition the stronger $W^{m, p}$-quasiconvexity condition (H3) we can deduce that $F[\cdot]$ is sequentially weakly lower semicontinuous on $W^{m, p}$ (see [43] for the case $m=1$; note that $W^{m, p}$-quasiconvexity is also a necessary condition for sequential weak lower semicontinuity on $W^{m, p}$ ). More precisely we can prove the following higher order version of [43], Theorem 4.4:

Theorem 1.3 (semicontinuity). Suppose that $f$ fulfills the q-growth condition (H1) with $1<p \leq q<\frac{n p}{n-1}$ and the $W^{m, p}\left(\Omega, \mathbb{R}^{N}\right)$-quasiconvexity condition $(\mathrm{H} 3)$. Then $F[\cdot]$ is sequentially weakly lower semicontinuous on $W^{m, p}\left(\Omega, \mathbb{R}^{N}\right)$.

Proof. As mentioned in [43], for $m=1$ the proof is basically identical to the proof of [21], Theorem 4.1, except for one difference. In Theorem 4.1 of $[21]$ the authors consider sequences of functions $\left(u_{k}\right)_{k}$ with $u_{k} \in W^{1, q}\left(\Omega, \mathbb{R}^{N}\right)$ and not general $W^{1, p}$-functions $u_{k}$. Therefore, on the other hand in [21] they do not have to assume $W^{1, p}\left(\Omega, \mathbb{R}^{N}\right)$ quasiconvexity for $f$ but only quasiconvexity. However, this does not change the proof in a significant way. For an improvement of the result in [21] see also [30].

In [17], Theorem 4.1, Esposito and Mingione have generalized Theorem 4.1 of [21] to higher orders under the condition $q<\frac{p m n}{m n-1}$.

The proof of our theorem follows beside slight differences exactly the proofs of [17], Theorem 4.1, and [21], Theorem 4.1. For the readers comfort we carry out the first step of the proof and give a sketch of the second one.

Step 1. We suppose that $\Omega=B:=B_{1}(0)$. Let $u, u_{k} \in W^{m, p}\left(B, \mathbb{R}^{N}\right), u_{k} \rightarrow u$ weakly in $W^{m, p}\left(B, \mathbb{R}^{N}\right)$ and let us further assume that $u$ is a polynomial function of degree $m, u=\sum_{|\alpha| \leq m} c_{\alpha} x^{\alpha}$ with $c_{\alpha} \in \mathbb{R}$. Since $u_{k} \rightarrow u$ in $W^{m, p}\left(B, \mathbb{R}^{N}\right)$, passing to a subsequence we may assume that

$$
\left\|u-u_{k}\right\|_{W^{m-1, p}\left(B, \mathbb{R}^{N}\right)} \leq k^{-1} .
$$


Let $\frac{1}{4}<r<1$ and $\rho=\frac{r+1}{2}$. Now instead of [17], Lemma 3.1, we apply here a variant of [10], Lemma 2.4, which in turn goes back on a theorem of Fonseca and Malý [22], Theorem 3.3. Due to [10], Lemma 2.4, there exist functions $z_{k} \in W^{m, p}\left(B, \mathbb{R}^{N}\right)$ and $r<s_{k}^{\prime}<r_{k}^{\prime}<\rho<1$ with $r_{k}^{\prime}$ and $s_{k}^{\prime}$ depending on $u$ and $u_{k}$, such that

$$
\begin{gathered}
z_{k}=u_{k} \quad \text { on } B_{s_{k}^{\prime}}, \quad z_{k}=u \quad \text { on } B \backslash B_{r_{k}^{\prime}}, \\
(\rho-r) k^{-1} \geq r_{k}^{\prime}-s_{k}^{\prime} \geq(\rho-r)(3 k)^{-1}
\end{gathered}
$$

and

$$
\begin{aligned}
\left\|D^{m} z_{k}\right\|_{L^{q}\left(B_{r_{k}^{\prime}} \backslash B_{s_{k}^{\prime}}, \mathbb{R}^{N}\right)} \leq & C \frac{(\rho-r)^{\tau}}{k^{\tau}}\left(\|u\|_{W^{m, p}\left(B_{\rho} \backslash B_{r}, \mathbb{R}^{N}\right)}+\left\|u_{k}\right\|_{W^{m, p}\left(B_{\rho} \backslash B_{r}, \mathbb{R}^{N}\right)}\right. \\
& \left.+\frac{k}{\rho-r}\left\|u-u_{k}\right\|_{W^{m-1, p}\left(B_{\rho} \backslash B_{r}, \mathbb{R}^{N}\right)}\right)
\end{aligned}
$$

where $C=C(n, p, q)$ and $\tau=\tau(n, p, q)>0$. Therefore, we have

$$
\mathcal{L}^{n}\left(B_{r_{k}^{\prime}} \backslash B_{s_{k}^{\prime}}\right) \leq \frac{c(r)}{k} \quad \text { and } \int_{B_{r_{k}^{\prime}} \backslash B_{s_{k}^{\prime}}}\left|D^{m} z_{k}\right|^{q} \mathrm{~d} x \leq c(r) k^{-q \tau} .
$$

Since $z_{k}-u \in W_{0}^{m, p}\left(B, \mathbb{R}^{N}\right)$ and $f$ is $W^{m, p}$-quasiconvex we can conclude that

$$
\int_{B} f\left(D^{m} u\right) \mathrm{d} x \leq \int_{B} f\left(D^{m} z_{k}\right) \mathrm{d} x .
$$

If we use the previous inequalities and the condition (H1) is follows that

$$
\begin{aligned}
\int_{B} f\left(D^{m} u\right) \mathrm{d} x-\int_{B} f\left(D^{m} u_{k}\right) \mathrm{d} x & \leq \int_{B}\left(f\left(D^{m} z_{k}\right)-f\left(D^{m} u_{k}\right)\right) \mathrm{d} x \\
& \leq \int_{B \backslash B_{r_{k}^{\prime}}} f\left(D^{m} u\right) \mathrm{d} x+\int_{B_{r_{k}^{\prime}} \backslash B_{s_{k}^{\prime}}} f\left(D^{m} z_{k}\right) \mathrm{d} x \\
& \leq c\left(\mathcal{L}^{n}\left(B \backslash B_{r_{k}^{\prime}}\right)+\int_{B_{r_{k}^{\prime}} \backslash B_{s_{k}^{\prime}}}\left(1+\left|D^{m} z_{k}\right|^{q}\right) \mathrm{d} x\right) \\
& \leq c(1-r)+c(r) k^{-q \tau}+c(r) k^{-1} .
\end{aligned}
$$

Letting first $k \rightarrow \infty$ and then $r \rightarrow 1$ we finally conclude

$$
\liminf _{k \rightarrow \infty} \int_{B} f\left(D^{m} u_{k}\right) \mathrm{d} x \geq \int_{B} f\left(D^{m} u\right) \mathrm{d} x .
$$

Step 2. Now we treat the general case. Let $u, u_{k} \in W^{m, p}\left(\Omega, \mathbb{R}^{N}\right), u_{k} \rightarrow u$ weakly in $W^{m, p}\left(\Omega, \mathbb{R}^{N}\right)$. This Step 2 is identical to Step 2 of [17], Theorem 4.1, and therefore, we will only give a brief sketch of it. Without loss of generality we may assume that

$$
\lim _{k \rightarrow \infty} \int_{\Omega} f\left(D^{m} u_{k}\right) \mathrm{d} x
$$


exists and is finite. There exist a non-negative Radon measure $\mu$ such that passing, if necessary, to a subsequence

$$
f\left(D^{m} u_{k}\right) \mathcal{L}^{n} \rightarrow \mu
$$

weakly in the sense of measures. Now using Step 1, it can be shown that

$$
\frac{\mathrm{d} \mu}{\mathrm{d} \mathcal{L}^{n}}\left(x_{0}\right) \geq f\left(D^{m} u\left(x_{0}\right)\right)
$$

for almost every $x_{0} \in \Omega$. Once (1.9) is verified the assertion of the theorem follows immediately. For further details we refer to [17].

Finally for $q \in\left[p, \frac{p n}{n-1}\right]$, Theorem 1.3 together with the coercivity assumption (H2) implies - using the direct method - the existence of at least one minimizer $u \in W^{m, p}\left(\Omega, \mathbb{R}^{N}\right)$ of $F[\cdot]$ in every Dirichlet class.

\section{Preliminary lemmas}

\subsection{The functions $V_{\beta}$ and $W_{\beta}$}

Let $\beta>0$. Throughout the paper we use the functions $V_{\beta}: \mathbb{R}^{k} \mapsto \mathbb{R}^{k}$ and $W_{\beta}: \mathbb{R}^{k} \mapsto \mathbb{R}^{k}$ which we define as

$$
V_{\beta}(\xi)=\left(1+|\xi|^{2}\right)^{\frac{\beta-2}{4}} \xi, \quad W_{\beta}(\xi)=(1+|\xi|)^{\frac{\beta}{2}-1} \xi
$$

for $\xi \in \mathbb{R}^{k}, k \in \mathbb{N}$. For $t \geq 0$ the functions $t \mapsto V_{\beta}(t)$ and $t \mapsto W_{\beta}(t)$ are both monotone non-decreasing. Using elementary inequalities we can deduce that

$$
c^{-1}(\beta)\left|W_{\beta}(\xi)\right| \leq\left|V_{\beta}(\xi)\right| \leq c(\beta)\left|W_{\beta}(\xi)\right|,
$$

see [16], Section 3. Further we can calculate that $\left|W_{p}\right|^{2}$ is convex for $1 \leq p<\infty$ and $\left|W_{p}\right|^{\frac{2}{p}}$ is convex only for $p \leq 2$ (in contrast to $\left|V_{p}\right|^{2}$ and $\left|V_{p}\right|^{\frac{2}{p}}$ ). In the following we present some useful properties of the functions $V_{\beta}$ and $W_{\beta}$.

Lemma 2.1. Let $1<p<\infty, \beta>0$ and $V_{\beta}, W_{\beta}: \mathbb{R}^{k} \rightarrow \mathbb{R}^{k}$ be the functions defined by (2.1). Then for any $\xi, \eta \in \mathbb{R}^{k}$ and $t>0$ we have:

(i) $\left|V_{p}(t \xi)\right| \leq \max \left\{t, t^{\frac{p}{2}}\right\}\left|V_{p}(\xi)\right|$;

(ii) $\quad\left|V_{\beta}(\xi+\eta)\right| \leq c(\beta)\left(\left|V_{\beta}(\xi)\right|+\left|V_{\beta}(\eta)\right|\right)$;

(iii) $\quad c^{-1}(k, p)|\xi-\eta| \leq \frac{\left|V_{p}(\xi)-V_{p}(\eta)\right|}{\left(1+|\xi|^{2}+|\eta|^{2}\right)^{\frac{p-2}{4}}} \leq c(k, p)|\xi-\eta|$;

(iv) $\quad c^{-1}(p, M)\left|V_{p}(\xi-\eta)\right| \leq\left|V_{p}(\xi)-V_{p}(\eta)\right| \leq c(k, p, M)\left|V_{p}(\xi-\eta)\right| \quad \forall \eta$ with $|\eta| \leq M$;

(v) $\quad\left(1+|\xi|^{2}+|\eta|^{2}\right)^{\frac{p}{2}} \leq c(p)\left(1+\left|V_{p}(\xi)\right|^{2}+\left|V_{p}(\eta)\right|^{2}\right)$.

Inequalities (i) and (ii) also hold if we replace $V$ by $W$.

Proof. The assertions (i)-(iv) for $V_{p}$ and $W_{p}$ can be found in [3], Lemma 2.1, [11], Lemma 2.1, and [16], Lemma 1, (v) is obvious.

The next lemma is taken from [2].

Lemma 2.2. Consider $f \in C^{2}$ satisfying (H1) and (H3s) and $A, B \in \bigodot^{m}\left(\mathbb{R}^{n}, \mathbb{R}^{N}\right)$ with $|A| \leq M+1$. Then the following estimates hold:

$$
\begin{aligned}
|f(A+B)-f(A)-D f(A) B| & \leq c\left|V_{q}(B)\right|^{2} \\
|D f(A+B)-D f(A)| & \leq c\left(1+|B|^{2}\right)^{\frac{q-2}{2}}|B|,
\end{aligned}
$$

where $c$ depends on $n, N, q, L, M$ and $K_{M}$. 


\subsection{A Poincaré-type inequality}

The following Poincaré-type inequality is for $p \geq 2$ a direct consequence of the usual Poincaré inequality. For $1<p<2$ it is a special case of the Sobolev-Poincaré-type inequality stated in [16] (see also [11]).

Lemma 2.3. Let $1<p<\infty$ and $V_{p}$ the function defined in (2.1). Then there exists a constant $c_{p}$ depending on $n, N$ and $p$ such that

$$
\int_{B_{\rho}\left(x_{0}\right)}\left|V_{p}\left(\frac{u-u_{x_{0}, \rho}}{\rho}\right)\right|^{2} \mathrm{~d} x \leq c_{p} \int_{B_{\rho}\left(x_{0}\right)}\left|V_{p}(D u)\right|^{2} \mathrm{~d} x
$$

for all $B_{\rho}\left(x_{0}\right) \subset \subset \mathbb{R}^{n}$ and every function $u \in W^{1, p}\left(B_{\rho}\left(x_{0}\right), \mathbb{R}^{N}\right)$.

\subsection{An iteration lemma}

The next lemma is a variant of [43], Lemma 6.6, [11], Lemma 2.7, and [16], Lemma 2, which in turn traces back to the well-known iteration lemma [24], Lemma 3.1, Chapter 5.

Lemma 2.4 (iteration lemma). Let $0 \leq \vartheta<1, A, B \geq 0, v \in W^{m, p}\left(B_{\rho}\left(x_{0}\right), \mathbb{R}^{N}\right), \alpha_{1}, \alpha_{2} \in \mathbb{R}$ and $g$ be $a$ non-negative bounded function satisfying

$$
g(r) \leq \vartheta g(s)+A \int_{B_{\rho}\left(x_{0}\right)} \sum_{k=0}^{m-1}\left|V_{p}\left(\frac{D^{k} v}{(s-r)^{m-k}}\right)\right|^{2} \mathrm{~d} x+B(s-r)^{\alpha_{1}}\left(\int_{B_{\rho}\left(x_{0}\right)} \sum_{k=0}^{m}\left|V_{p}\left(\frac{D^{k} v}{(s-r)^{m-k}}\right)\right|^{2} \mathrm{~d} x\right)^{\alpha_{2}}
$$

for all $\rho / 2 \leq r<s \leq \rho$. Then there exists a constant $c=c\left(\vartheta, p, \alpha_{1}, \alpha_{2}\right)$ such that

$$
g(\rho / 2) \leq c\left[A \int_{B_{\rho}\left(x_{0}\right)} \sum_{k=0}^{m-1}\left|V_{p}\left(\frac{D^{k} v}{\rho^{m-k}}\right)\right|^{2} \mathrm{~d} x+B \rho^{\alpha_{1}}\left(\int_{B_{\rho}\left(x_{0}\right)} \sum_{k=0}^{m}\left|V_{p}\left(\frac{D^{k} v}{\rho^{m-k}}\right)\right|^{2} \mathrm{~d} x\right)^{\alpha_{2}}\right] .
$$

\subsection{Trace preserving operator}

The trace preserving operator $T$ was first introduced in [21] and later used in [9]. A version for higher derivatives can be found in [22], Theorem 3.3. We state the extension result here in a slightly changed form.

Lemma 2.5. Let $\tilde{\Omega} \subset \mathbb{R}^{n}$ be a bounded domain. Let $u \in W^{m, p}\left(\tilde{\Omega}, \mathbb{R}^{N}\right), 1 \leq p<\infty, 0<r<s$ and $B_{s} \subset \tilde{\Omega}$. Then there exists a bounded linear operator

$$
T_{r, s}: W^{m, p}(\tilde{\Omega}) \rightarrow W^{m, p}(\tilde{\Omega})
$$

with the following properties:

(i) $u=T_{r, s} u$ a.e. on $\left(\tilde{\Omega} \backslash B_{s}\right) \cup B_{r}$ and $T_{r, s} u-u \in W_{0}^{m, p}\left(B_{s} \backslash \overline{B_{r}}, \mathbb{R}^{N}\right)$;

(ii) $\left\|D^{k} T_{r, s} u\right\|_{L^{p}\left(B_{s} \backslash B_{r}, \mathbb{R}^{N}\right)} \leq c(n, m, p)\left\|D^{k} u\right\|_{L^{p}\left(B_{s} \backslash B_{r}, \mathbb{R}^{N}\right)}$ for $k=0,1, \ldots, m$;

(iii) $\left\|D^{k} T_{r, s} u\right\|_{L^{q}\left(B_{s} \backslash B_{r}, \mathbb{R}^{N}\right)}$

$$
\leq c(n, m, p, q)(s-r)^{\frac{n}{q}-\frac{n-1}{p}} \cdot\left(\sup _{t \in] r, s[}(t-r)^{-1} \int_{B_{t} \backslash B_{r}}\left|D^{k} u\right|^{p} \mathrm{~d} x+\sup _{t \in] r, s[}(s-t)^{-1} \int_{B_{s} \backslash B_{t}}\left|D^{k} u\right|^{p} \mathrm{~d} x\right)^{\frac{1}{p}}
$$

for $k=0,1, \ldots, m$ and for all $1 \leq p \leq q<\frac{n}{n-1} p ;$

(iv) $\left\|\left|V_{p}\left(D^{k} T_{r, s} u\right)\right|^{2}\right\|_{L^{1}\left(B_{s} \backslash B_{r}, \mathbb{R}^{N}\right)} \leq c(n, m, p)\left\|\left|V_{p}\left(D^{k} u\right)\right|^{2}\right\|_{L^{1}\left(B_{s} \backslash B_{r}, \mathbb{R}^{N}\right)} \quad$ for $k=0,1, \ldots, m$; 
(v) $\left\|\left|V_{p}\left(D^{k} T_{r, s} u\right)\right|^{2}\right\|_{L^{\tilde{q}}\left(B_{s} \backslash B_{r}, \mathbb{R}^{N}\right)}$

$$
\leq c(n, m, p, q)(s-r)^{\frac{n}{q}-(n-1)} \cdot\left(\sup _{t \in] r, s[}(t-r)^{-1} \int_{B_{t} \backslash B_{r}}\left|V_{p}\left(D^{k} u\right)\right|^{2} \mathrm{~d} x+\sup _{t \in] r, s[}(s-t)^{-1} \int_{B_{s} \backslash B_{t}}\left|V_{p}\left(D^{k} u\right)\right|^{2} \mathrm{~d} x\right)
$$

for $k=0,1, \ldots, m$ and for all $1 \leq \tilde{q}<\frac{n}{n-1}$.

Proof. As mentioned above this lemma can be found in [22], Theorem 3.3, in a slightly different form and (i)-(iii) follow from it directly by setting $\Omega=B_{s} \backslash \overline{B_{r}}$ and $\varepsilon=\frac{r-s}{2}$. Since $c^{-1}\left|W_{p}\right| \leq\left|V_{p}\right| \leq c\left|W_{p}\right|$, it suffices to show (iv) and (v) for $W_{p}$ instead of $V_{p}$ and therefore we can exploit the convexity of $\left|W_{p}\right|^{2}$. In [22], Theorem 3.3, the following estimate is stated:

$$
\left|D^{k} T_{r, s} u(x)\right| \leq C \underset{B_{3 r_{i}}\left(x_{i}\right)}{f}\left|D^{k} u\right| \mathrm{d} y \quad \text { for } x \in B_{r_{i}}\left(x_{i}\right), \quad B_{r_{i}}\left(x_{i}\right) \in \mathcal{W} \quad k=0,1, \ldots, m,
$$

where $\mathcal{W}$ denotes a family of Whitney balls for $\Omega=B_{s} \backslash \overline{B_{r}}$. Then estimate (T1) together with Jensen's inequality and Lemma 2.1 gives us the following inequality:

$$
\begin{aligned}
f_{B_{r_{i}}\left(x_{i}\right)}\left|W_{p}\left(D^{k} T_{r, s} u(x)\right)\right|^{2 \tilde{q}} \mathrm{~d} x & \leq c \underset{B_{r_{i}}\left(x_{i}\right)}{f}\left|W_{p}\left(\underset{B_{3 r_{i}}\left(x_{i}\right)}{f}\left|D^{k} u\right| \mathrm{d} y\right)\right|^{2 \tilde{q}} \mathrm{~d} x \\
& \leq c\left(\underset{B_{3 r_{i}\left(x_{i}\right)}}{f}\left|W_{p}\left(D^{k} u\right)\right|^{2} \mathrm{~d} y\right)^{\tilde{q}} .
\end{aligned}
$$

If we use the last inequality instead of (T2) in [22], Theorem 3.3, and proceed otherwise as for the derivation of (T3) and (T5) in [22], Theorem 3.3, we can deduce (iv) and (v).

Later we want to estimate the terms on the right hand side of Lemma 2.5 (iii) and (v) using the following lemma. A proof of this lemma is given e.g. in [21].

Lemma 2.6. Let $-\infty<r<s<\infty$ and a continuous nondecreasing function $\Xi:[r, s] \rightarrow \mathbb{R}$ be given. Then there are $\tilde{r} \in\left[r, \frac{2 r+s}{3}\right]$ and $\tilde{s} \in\left[\frac{r+2 s}{3}, s\right]$ for which hold:

$$
\begin{aligned}
& \frac{\Xi(t)-\Xi(\tilde{r})}{t-\tilde{r}} \leq 3 \frac{\Xi(s)-\Xi(r)}{s-r} \\
& \frac{\Xi(\tilde{s})-\Xi(t)}{\tilde{s}-t} \leq 3 \frac{\Xi(s)-\Xi(r)}{s-r},
\end{aligned}
$$

for every $t \in] \tilde{r}, \tilde{s}[$. In particular we have

$$
\frac{s-r}{3} \leq \tilde{s}-\tilde{r} \leq s-r .
$$

\section{Caccioppoli inequality}

Similarly to the case $m=1$ treated in [43], we use the trace operator $T$ defined in Lemma 2.5 to prove a Caccioppoli-type inequality, which differs from the usual Caccioppoli inequality under standard growth conditions (see e.g. [16,32] for higher orders) by an additional term on the right hand side. Using common smallness assumptions, we will see that this additional term causes no problems when deriving the decay estimate for the excess function $\Phi(\rho)$ (see Sect. 6). 
Lemma 3.1 (Caccioppoli inequality). Let $1<p \leq q<\min \left\{p+\frac{1}{n}, \frac{2 n-1}{2 n-2} p\right\}$, i.e. $q$ satisfies (1.6), and $M>0$. We consider a $W^{m, p}$-minimizer $u$ of the functional $F[u]=\int_{\Omega} f\left(D^{m} u\right)$, where the integrand $f \in C^{2}$ fulfills (H1) and (H3s). Then for all balls $B_{\rho} \subset \subset \Omega$ and for all polynomials $P: \Omega \rightarrow \mathbb{R}^{N}$ of degree $\leq m$ with $\left|D^{m} P\right| \leq M+1$ we have

$$
f_{B_{\rho / 2}}\left|V_{p}\left(D^{m} v\right)\right|^{2} \mathrm{~d} x \leq c_{c}\left[f_{B_{\rho}}^{m-1} \sum_{k=0}^{m}\left|V_{p}\left(\frac{D^{k} v}{\rho^{m-k}}\right)\right|^{2} \mathrm{~d} x+\left(f_{B_{\rho}} \sum_{k=0}^{m}\left|V_{p}\left(\frac{D^{k} v}{\rho^{m-k}}\right)\right|^{2} \mathrm{~d} x\right)^{\frac{n}{n-1}}\right],
$$

where we have set $v=u-P$. The constant $c_{c}$ is of the form $c_{c}\left(n, N, m, p, q, L, M, \lambda_{M}, K_{M}\right)$.

Proof. Choose $\frac{\rho}{2} \leq r<s \leq \rho$ and set

$$
\Xi(t):=\int_{B_{t}} \sum_{k=0}^{m}\left|V_{p}\left(\frac{D^{k} v}{(s-r)^{m-k}}\right)\right|^{2} \mathrm{~d} x .
$$

For this function $\Xi$ we choose $r \leq \tilde{r}<\tilde{s} \leq s$ as in Lemma 2.6. Now we define a cut-off function $\eta \in C_{0}^{\infty}\left(B_{\rho}\right)$ which satisfies $0 \leq \eta \leq 1, \eta \equiv 1$ in a neighborhood of $\overline{B_{\tilde{r}}}, \eta \equiv 0$ on $B_{\rho} \backslash B_{\tilde{s}}$ and $\left|D^{k} \eta\right| \leq\left(c_{\eta}(\tilde{s}-\tilde{r})\right)^{-k}$ for all $1 \leq k \leq m$. Using the operator $T$ from Lemma 2.5 we define

$$
\phi:=(1-\eta) v, \quad \psi:=T_{\tilde{r}, \tilde{s}} \phi \quad \text { and } \quad \varphi:=v-\psi,
$$

where

$$
D^{m} u-D^{m} P=D^{m} v=D^{m} \varphi+D^{m} \psi \quad \text { on } B_{\rho} .
$$

According to (i) of Lemma 2.5 we have $\varphi \in W_{0}^{m, p}\left(B_{\tilde{s}}, \mathbb{R}^{N}\right)$ and $\varphi=v$ on $B_{\tilde{r}}$. With the product formula and (2.5) we calculate

$$
\left|D^{m} \phi\right|=\left|\sum_{k=0}^{m}\left(\begin{array}{c}
m \\
k
\end{array}\right) D^{m-k}(1-\eta) \otimes D^{k} v\right| \leq c \sum_{k=0}^{m} \frac{\left|D^{k} v\right|}{(s-r)^{m-k}} .
$$

As abbreviation we further set

$$
X:=\int_{B_{s} \backslash B_{r}} \sum_{k=0}^{m}\left|V_{p}\left(\frac{D^{k} v}{(s-r)^{m-k}}\right)\right|^{2} \mathrm{~d} x .
$$

First we derive some useful inequalities. If we apply (vi) and (v) of Lemma 2.5 to our operator $T_{\tilde{r}, \tilde{s}}$ and use then Lemma 2.1 (i), (ii) and inequality (3.2) we get the following estimates:

$$
\int_{B_{\tilde{s}} \backslash B_{\tilde{r}}}\left|V_{p}\left(D^{m} T_{\tilde{s}, \tilde{r}} \phi\right)\right|^{2} \leq c \int_{B_{\tilde{s}} \backslash B_{\tilde{r}}}\left|V_{p}\left(D^{m} \phi\right)\right|^{2} \mathrm{~d} x \leq c X
$$

and

$$
\begin{aligned}
\int_{B_{\tilde{s} \backslash B_{\tilde{r}}}\left|V_{p}\left(D^{m} T_{\tilde{s}, \tilde{r}} \phi\right)\right|^{2 \tilde{q}} \leq} & c(\tilde{s}-\tilde{r})^{n}\left(\sup _{t \in[\tilde{r}, \tilde{s}[} \frac{(\tilde{s}-\tilde{r})^{1-n}}{(t-\tilde{r})} \int_{B_{t} \backslash B_{\tilde{r}}}\left|V_{p}\left(D^{m} \phi\right)\right|^{2} \mathrm{~d} x\right. \\
& \left.+\sup _{t \in] \tilde{r}, \tilde{s}[} \frac{(\tilde{s}-\tilde{r})^{1-n}}{(\tilde{s}-t)} \int_{B_{\tilde{s} \backslash B_{t}}}\left|V_{p}\left(D^{m} \phi\right)\right|^{2} \mathrm{~d} x\right)^{\tilde{q}} \\
\leq & c(\tilde{s}-\tilde{r})^{n}\left(\sup _{t \in] \tilde{r}, \tilde{s}[}(\tilde{s}-\tilde{r})^{1-n} \frac{\Xi(t)-\Xi(\tilde{r})}{(t-\tilde{r})}+\sup _{t \in] \tilde{r}, \tilde{s}[}(\tilde{s}-\tilde{r})^{1-n} \frac{\Xi(\tilde{s})-\Xi(t)}{(\tilde{s}-t)}\right)^{\tilde{q}}
\end{aligned}
$$


for $1 \leq \tilde{q}<\frac{n}{n-1}$. Combining the last inequality with the estimates of Lemma 2.6 we obtain

$$
\int_{B_{\tilde{s}} \backslash B_{\tilde{r}}}\left|V_{p}\left(D^{m} T_{\tilde{s}, \tilde{r}} \phi\right)\right|^{2 \tilde{q}} \leq c(s-r)^{n}\left(\frac{X}{(s-r)^{n}}\right)^{\tilde{q}}, \quad \text { for } 1 \leq \tilde{q}<\frac{n}{n-1} .
$$

We remark that for $\tilde{q}=1$ this formula is identical to equation (3.3).

Now, using the quasiconvexity condition (H3s) we get

$$
\begin{aligned}
\lambda_{M} \int_{B_{\tilde{r}}}\left|V_{p}\left(D^{m} v\right)\right|^{2} \mathrm{~d} x= & \lambda_{M} \int_{B_{\tilde{r}}}\left|V_{p}\left(D^{m} \varphi\right)\right|^{2} \mathrm{~d} x \\
\leq & \lambda_{M} \int_{B_{\tilde{s}}}\left(1+\left|D^{m} \varphi\right|^{2}\right)^{\frac{p-2}{2}}\left|D^{m} \varphi\right|^{2} \mathrm{~d} x \leq \int_{B_{\tilde{s}}}\left(f\left(D^{m} P+D^{m} \varphi\right)-f\left(D^{m} P\right)\right) \mathrm{d} x \\
= & \int_{B_{\tilde{s}}}\left(f\left(D^{m} u-D^{m} \psi\right)-f\left(D^{m} u\right)\right) \mathrm{d} x+\int_{B_{\tilde{s}}}\left(f\left(D^{m} u\right)-f\left(D^{m} u-D^{m} \varphi\right)\right) \mathrm{d} x \\
& +\int_{B_{\tilde{s}}}\left(f\left(D^{m} P+D^{m} \psi\right)-f\left(D^{m} P\right)\right) \mathrm{d} x .
\end{aligned}
$$

The second term is smaller or equal to zero because of the minimality of $u$. Therefore we deduce with Lemmas 2.2 and 2.1 (i), (ii) (for $\beta=2 q-2$ )

$$
\begin{aligned}
\lambda_{M} \int_{B_{r}}\left|V_{p}\left(D^{m} v\right)\right|^{2} \mathrm{~d} x \leq & \int_{B_{\tilde{s}}}\left[\int_{0}^{1}\left(D f\left(D^{m} P\right)-D f\left(D^{m} u-\tau D^{m} \psi\right)\right) \mathrm{d} \tau D^{m} \psi\right. \\
& \left.+f\left(D^{m} P+D^{m} \psi\right)-f\left(D^{m} P\right)-D f\left(D^{m} P\right) D^{m} \psi\right] \mathrm{d} x \\
\leq & c \int_{B_{\tilde{s}}}\left[\int_{0}^{1}\left(1+\left|D^{m} v-\tau D^{m} \psi\right|^{2}\right)^{\frac{q-2}{2}}\left|D^{m} v-\tau D^{m} \psi\right| \mathrm{d} \tau\left|D^{m} \psi\right|+\left|V_{q}\left(D^{m} \psi\right)\right|^{2}\right] \mathrm{d} x \\
\leq & c \int_{B_{\tilde{s}} \backslash B_{\tilde{r}}}\left[\left|V_{q}\left(D^{m} \psi\right)\right|^{2}+\left(1+\left|D^{m} v\right|^{2}\right)^{\frac{q-2}{2}}\left|D^{m} v\right|\left|D^{m} \psi\right|\right] \mathrm{d} x \\
= & c[(I)+(I I)],
\end{aligned}
$$

where $c$ is a constant depending on $n, N, p, q, M, K_{M}$ and $L$.

Estimate for $(I)$. To estimate $(I)$ we apply inequality (v) of Lemma 2.1 and deduce

$$
\begin{aligned}
(I) & =c \int_{B_{\tilde{s}} \backslash B_{\tilde{r}}}\left|V_{p}\left(D^{m} \psi\right)\right|^{2}\left(1+\left|D^{m} \psi\right|^{2}\right)^{\frac{p}{2} \frac{q-p}{p}} \mathrm{~d} x \\
& \leq c \int_{B_{\tilde{s}} \backslash B_{\tilde{r}}}\left|V_{p}\left(D^{m} \psi\right)\right|^{2}\left(1+\left|V_{p}\left(D^{m} \psi\right)\right|^{2}\right)^{\frac{q-p}{p}} \mathrm{~d} x \\
& \leq c \int_{B_{\tilde{s}} \backslash B_{\tilde{r}}}\left(\left|V_{p}\left(D^{m} \psi\right)\right|^{2}+\left|V_{p}\left(D^{m} \psi\right)\right|^{\frac{2 q}{p}}\right) \mathrm{d} x .
\end{aligned}
$$


The first term on the right-hand side of the preceding inequality can be estimated using (3.3) (recall $\left.\psi:=T_{\tilde{r}, \tilde{s}} \phi\right)$, while the second one is treated using inequality (3.4) with $\tilde{q}=\frac{q}{p}$ (recall that $q<\frac{n p}{n-1}$ ). Altogether we get

$$
(I) \leq c\left[X+(s-r)^{n}\left(\frac{X}{(s-r)^{n}}\right)^{\frac{q}{p}}\right] \leq c\left[X+(s-r)^{n}\left(\frac{X}{(s-r)^{n}}\right)^{\frac{n}{n-1}}\right]
$$

where $c=c(n, N, m, p, q)$.

Estimate for $(I I)$. For the estimation of $(I I)$ we distinguish the cases $p>2 \frac{n-1}{n}$ and $1<p \leq 2 \frac{n-1}{n}$.

Case 1: $1<p \leq 2 \frac{n-1}{n}$. In this case (1.6) reads $q<p \frac{2 n-1}{2 n-2}$ and therefore we have also $q<\frac{p}{2}+1$. We estimate the integrand of $(I I)$ pointwise. If $\left|D^{m} v\right| \leq\left|D^{m} \psi\right|$ we use that $\left(1+t^{2}\right)^{\frac{q-2}{2}} t$ is non-decreasing in $t \geq 0$ to get

$$
\left(1+\left|D^{m} v\right|^{2}\right)^{\frac{q-2}{2}}\left|D^{m} v\right|\left|D^{m} \psi\right| \leq\left|V_{q}\left(D^{m} \psi\right)\right|^{2}
$$

and if $\left|D^{m} v\right|>\left|D^{m} \psi\right|$ we obtain (recall $2 q-p-1<0$ )

$$
\begin{aligned}
\left(1+\left|D^{m} v\right|^{2}\right)^{\frac{q-2}{2}}\left|D^{m} v \| D^{m} \psi\right| & =\left(1+\left|D^{m} v\right|^{2}\right)^{\frac{p-2}{4}}\left(1+\left|D^{m} v\right|^{2}\right)^{\frac{2 q-p-2}{4}}\left|D^{m} v\right|\left|D^{m} \psi\right| \\
& \leq\left|V_{p}\left(D^{m} v\right)\right|\left|V_{2 q-p}\left(D^{m} \psi\right)\right| .
\end{aligned}
$$

These pointwise estimates together with Hölder's inequality imply the following estimate for $(I I)$ :

$$
\begin{aligned}
& (I I) \leq \int_{B_{\tilde{s}} \backslash B_{\tilde{r}}}\left(\left|V_{q}\left(D^{m} \psi\right)\right|^{2}+\left|V_{p}\left(D^{m} v\right)\right|\left|V_{2 q-p}\left(D^{m} \psi\right)\right|\right) \mathrm{d} x \\
& \leq \int_{B_{\tilde{s}} \backslash B_{\tilde{r}}}\left|V_{q}\left(D^{m} \psi\right)\right|^{2} \mathrm{~d} x+\left(\int_{B_{\tilde{S}} \backslash B_{\tilde{r}}}\left|V_{2 q-p}\left(D^{m} \psi\right)\right|^{2} \mathrm{~d} x\right)^{\frac{1}{2}}\left(\int_{B_{\tilde{s}} \backslash B_{\tilde{r}}}\left|V_{p}\left(D^{m} v\right)\right|^{2} \mathrm{~d} x\right)^{\frac{1}{2}} .
\end{aligned}
$$

We have already estimated the first term (see estimation of $(I)$ ). The second integral can be estimated analogously recalling that $q<p \frac{2 n-1}{2 n-2}$ :

$$
\int_{B \tilde{s} \backslash B_{\tilde{r}}}\left|V_{2 q-p}\left(D^{m} \psi\right)\right|^{2} \mathrm{~d} x \leq c X+c(s-r)^{n}\left(\frac{X}{(s-r)^{n}}\right)^{2 \frac{q}{p}-1} \leq c\left[X+(s-r)^{n}\left(\frac{X}{(s-r)^{n}}\right)^{\frac{n}{n-1}}\right] .
$$

Collecting terms we deduce

$$
(I I) \leq c\left[X+(s-r)^{n}\left(\frac{X}{(s-r)^{n}}\right)^{\frac{n}{n-1}}\right]
$$

with $c=c(n, N, m, p, q)$.

Case 2: $p>2 \frac{n-1}{n}$. In this case (1.6) reads $q<p+\frac{1}{n}$ and we have $\frac{p}{2}+1<p+\frac{1}{n}$. If $p \leq q \leq \frac{p}{2}+1$ we can estimate $(I I)$ exactly as in the Case 1. Otherwise $q$ satisfies $2 q-p-2>0$ and applying Young's inequality we estimate the integrand of $(I I)$ for $\left|D^{m} v\right| \leq 1$ by

$$
\begin{aligned}
\left(1+\left|D^{m} v\right|^{2}\right)^{\frac{q-2}{2}}\left|D^{m} v\right|\left|D^{m} \psi\right| & =\left(1+\left|D^{m} v\right|^{2}\right)^{\frac{p-2}{4}+\frac{2 q-p-2}{4}}\left|D^{m} v\right|\left|D^{m} \psi\right| \\
& \leq c\left(1+\left|D^{m} v\right|^{2}\right)^{\frac{p-2}{4}}\left|D^{m} v\right|\left|D^{m} \psi\right| \\
& \leq c(p, q)\left(\left|V_{p}\left(D^{m} v\right)\right|^{2}+\left|D^{m} \psi\right|^{2}\right),
\end{aligned}
$$


and for $\left|D^{m} v\right|>1$ by

$$
\begin{aligned}
\left(1+\left|D^{m} v\right|^{2}\right)^{\frac{q-2}{2}}\left|D^{m} v\right|\left|D^{m} \psi\right| & =\left(1+\left|D^{m} v\right|^{2}\right)^{\frac{p-2}{2} \frac{q-1}{p}+\frac{2 q-p-2}{2 p}}\left|D^{m} v\right|\left|D^{m} \psi\right| \\
& \leq c\left(1+\left|D^{m} v\right|^{2}\right)^{\frac{p-2}{2} \frac{q-1}{p}}\left|D^{m} v\right|^{\frac{q-1}{p}}\left|D^{m} \psi\right| \\
& \leq c(p, q)\left(\left|V_{p}\left(D^{m} v\right)\right|^{2}+\left|D^{m} \psi\right|^{\frac{p}{p+1-q}}\right) .
\end{aligned}
$$

Thus, since $\frac{p}{p+1-q} \geq 2$ this pointwise estimates lead us to

$$
\begin{aligned}
(I I) & \leq c \int_{B_{\tilde{s} \backslash B_{\tilde{r}}}}\left(\left|V_{p}\left(D^{m} v\right)\right|^{2}+\left|D^{m} \psi\right|^{2}+\left|D^{m} \psi\right|^{\frac{p}{p+1-q}}\right) \mathrm{d} x \\
& \leq c\left(X+\int_{B_{\tilde{s} \backslash B_{\tilde{r}}}}\left(\left|V_{p}\left(D^{m} \psi\right)\right|^{2}+\left|V_{p}\left(D^{m} \psi\right)\right|^{\frac{2}{p+1-q}}\right) \mathrm{d} x\right) .
\end{aligned}
$$

We use the estimates (3.3) and (3.4) (remember that $q<p+\frac{1}{n}$ ) to get

$$
(I I) \leq c\left[X+(s-r)^{n}\left(\frac{X}{(s-r)^{n}}\right)^{\frac{1}{p+1-q}}\right] \leq c\left[X+(s-r)^{n}\left(\frac{X}{(s-r)^{n}}\right)^{\frac{n}{n-1}}\right]
$$

where $c=c(n, N, m, p, q)$.

Inserting the preceding estimates for $(I)$ and $(I I)$ into (3.5) we finally arrive at

$$
\int_{B_{r}}\left|V_{p}\left(D^{m} v\right)\right|^{2} \mathrm{~d} x \leq C_{1} X+C_{1}(s-r)^{n}\left(\frac{X}{(s-r)^{n}}\right)^{\frac{n}{n-1}}
$$

with $C_{1}=C_{1}\left(n, N, m, p, q, L, K_{M}, \lambda_{M}, M\right)$. Adding $C_{1} \int_{B_{r}}\left|V_{p}\left(D^{m} v\right)\right|^{2}$ on both sides leads to

$$
\begin{aligned}
\int_{B_{r}}\left|V_{p}\left(D^{m} v\right)\right|^{2} \mathrm{~d} x \leq & \frac{C_{1}}{1+C_{1}} \int_{B_{s}}\left|V_{p}\left(D^{m} v\right)\right|^{2} \mathrm{~d} x+\int_{B_{\rho}} \sum_{k=0}^{m-1}\left|V_{p}\left(\frac{D^{k} v}{(s-r)^{m-k}}\right)\right|^{2} \mathrm{~d} x \\
& +(s-r)^{n}\left(\frac{1}{(s-r)^{n}} \int_{B_{\rho}} \sum_{k=0}^{m}\left|V_{p}\left(\frac{D^{k} v}{(s-r)^{m-k}}\right)\right|^{2} \mathrm{~d} x\right)^{\frac{n}{n-1}} .
\end{aligned}
$$

Now we can apply the iteration Lemma 2.4 with $\vartheta=\frac{C_{1}}{1+C_{1}}$ and $g(t)=\int_{B_{t}}\left|V_{p}\left(D^{m} v\right)\right|^{2} \mathrm{~d} x$ to infer the desired estimate:

$$
\underset{B_{\rho / 2}}{f}\left|V_{p}\left(D^{m} v\right)\right|^{2} \mathrm{~d} x \leq c\left[f_{B_{\rho}}^{m-1} \sum_{k=0}\left|V_{p}\left(\frac{D^{k} v}{\rho^{m-k}}\right)\right|^{2} \mathrm{~d} x+\left(f_{B_{\rho}} \sum_{k=0}^{m}\left|V_{p}\left(\frac{D^{k} v}{\rho^{m-k}}\right)\right|^{2} \mathrm{~d} x\right)^{\frac{n}{n-1}}\right],
$$

with $c=c\left(n, N, m, p, q, L, K_{M}, \lambda_{M}, M\right)$.

\section{4. $\mathcal{A}$-harmonic approximation}

In this section we consider a bilinear form $\mathcal{A} \in \bigodot^{2}\left(\bigodot^{m}\left(\mathbb{R}^{n}, \mathbb{R}^{N}\right), \mathbb{R}\right)$ which is elliptic in the sense of LegendreHadamard with ellipticity constant $\kappa$ and upper bound $K$, that means

$$
\mathcal{A}\left(\xi^{m} \otimes \eta, \xi^{m} \otimes \eta\right) \geq \kappa|\xi|^{2 m}|\eta|^{2}
$$


for all $\xi \in \mathbb{R}^{n}$ and $\eta \in \mathbb{R}^{N}$ and

$$
\mathcal{A}(A, B) \leq K|A||B| \quad \forall A, B \in \bigodot^{m}\left(\mathbb{R}^{n}, \mathbb{R}^{N}\right)
$$

The next lemma provides a standard a-priori estimate for solutions of linear elliptic systems with constant coefficients.

Lemma 4.1. Consider $\mathcal{A}$ satisfying (4.1) and (4.2) and a function $h \in W^{m, 1}\left(B_{\rho}\left(x_{0}\right), \mathbb{R}^{N}\right)$ with

$$
\int_{B_{\rho}\left(x_{0}\right)} \mathcal{A}\left(D^{m} h, D^{m} \varphi\right) \mathrm{d} x=0
$$

for all $\varphi \in C_{0}^{\infty}\left(B_{\rho}\left(x_{0}\right), \mathbb{R}^{N}\right)$. Then $h \in C^{\infty}\left(B_{\rho}\left(x_{0}\right), \mathbb{R}^{N}\right)$ and there exists a constant $c_{a}=c_{a}(n, N, m, \kappa, K)$ such that the following estimate holds:

$$
\sup _{B_{\rho / 2}\left(x_{0}\right)}\left|D^{m} h\right|+\rho \sup _{B_{\rho / 2}\left(x_{0}\right)}\left|D^{m+1} h\right| \leq c_{a} f_{B_{\rho}\left(x_{0}\right)}\left|D^{m} h\right| \mathrm{d} x .
$$

Proof. This lemma can be found in [32] for $h \in W^{m, 2}$. A standard approximation argument (see [11], Prop. 2.1) shows the desired estimates for $h \in W^{m, 1}$.

The main tool for proving partial regularity via $\mathcal{A}$-harmonic approximation is the so called $\mathcal{A}$-harmonic approximation lemma. For $m=1$ a basic version of this lemma is given in [14]. In our case we need the following higher order version:

Lemma $4.2((\mathcal{A}, m)$-harmonic approximation lemma). Let $\kappa, K$ be positive constants and $1<\tilde{p} \leq 2$. Then for any $\varepsilon>0$ there exists $\delta=\delta(n, N, m, \kappa, K, \varepsilon) \in(0,1]$ with the following property: for any given $\mathcal{A}$ satisfying $(4.1)$ and (4.2) and for any $v \in W^{m, \tilde{p}}\left(B_{\rho}\left(x_{0}\right), \mathbb{R}^{N}\right)$ with

$$
\begin{gathered}
f_{B_{\rho}\left(x_{0}\right)}\left|W_{\tilde{p}}\left(D^{m} v\right)\right|^{2} \mathrm{~d} x \leq \gamma^{2} \leq 1 \quad \text { and } \\
\left|f_{B_{\rho}\left(x_{0}\right)} \mathcal{A}\left(D^{m} v, D^{m} \varphi\right) \mathrm{d} x\right| \leq \gamma \delta \sup _{B_{\rho}\left(x_{0}\right)}\left|D^{m} \varphi\right| \quad \forall \varphi \in C_{0}^{\infty}\left(B_{\rho}\left(x_{0}\right), \mathbb{R}^{N}\right),
\end{gathered}
$$

there exists an $(\mathcal{A}, m)$-harmonic function $h \in W^{m, \tilde{p}}\left(B_{\rho}\left(x_{0}\right), \mathbb{R}^{N}\right)$ satisfying

$$
f_{B_{\rho}\left(x_{0}\right)}\left|W_{\tilde{p}}\left(D^{m} h\right)\right|^{2} \mathrm{~d} x \leq 1 \quad \text { and } \quad \int_{B_{\rho}\left(x_{0}\right)} \sum_{k=0}^{m-1}\left|W_{\tilde{p}}\left(\frac{D^{k}(v-\gamma h)}{\rho}\right)\right|^{2} \mathrm{~d} x \leq \gamma^{2} \varepsilon
$$

A function $h$ is called $(\mathcal{A}, m)$-harmonic if $\int_{B_{\rho}\left(x_{0}\right)} \mathcal{A}\left(D^{m} h, D^{m} \varphi\right) \mathrm{d} x=0$ for all $\varphi \in C_{0}^{\infty}\left(B_{\rho}\left(x_{0}\right), \mathbb{R}^{N}\right)$.

Proof. For $\tilde{p}=2$ a proof of this lemma is presented in [32]. In the case $1<\tilde{p}<2$ the lemma is shown in [16], Lemma 6 , for $m=1$. The here stated higher order version is an easy adaption of [16], Lemma 6 .

Since we want to treat the cases $p \geq 2$ and $1<p<2$ simultaneously it is useful to combine the last two lemmas and state them here in the form of [43], Lemma 6.8. 
Corollary 4.3. Let $\kappa, K$ be positive constants and $1<p<\infty$. Then for any $\varepsilon>0$ there exists $\delta=$ $\delta(n, N, m, \kappa, K, \varepsilon) \in(0,1]$ with the following property: for any given $\mathcal{A}$ satisfying (4.1) and (4.2) and for any $v \in W^{m, p}\left(B_{\rho}\left(x_{0}\right), \mathbb{R}^{N}\right)$ with

$$
\begin{gathered}
f_{B_{\rho}\left(x_{0}\right)}\left|V_{p}\left(D^{m} v\right)\right|^{2} \mathrm{~d} x \leq \gamma^{2} \leq 1 \quad \text { and } \\
\left|f_{B_{\rho}\left(x_{0}\right)} \mathcal{A}\left(D^{m} v, D^{m} \varphi\right) \mathrm{d} x\right| \leq \gamma \delta \sup _{B_{\rho}\left(x_{0}\right)}\left|D^{m} \varphi\right| \quad \forall \varphi \in C_{0}^{\infty}\left(B_{\rho}\left(x_{0}\right), \mathbb{R}^{N}\right)
\end{gathered}
$$

exists an $(\mathcal{A}, m)$-harmonic function $h \in C^{\infty}\left(B_{\rho}\left(x_{0}\right), \mathbb{R}^{N}\right)$ with

$$
\sup _{B_{\rho / 2}\left(x_{0}\right)}\left|D^{m} h\right|+\rho \sup _{B_{\rho / 2}\left(x_{0}\right)}\left|D^{m+1} h\right| \leq c_{a} \quad \text { and }{\underset{B}{B_{\rho / 2}\left(x_{0}\right)}}_{f} \sum_{k=0}^{m-1}\left|V_{p}\left(\frac{D^{k}(v-\gamma h)}{\rho}\right)\right|^{2} \mathrm{~d} x \leq \gamma^{2} \varepsilon,
$$

where $c_{a}=c_{a}(n, N, m, \kappa, K)$ denotes the constant of Lemma 4.1.

Proof. In the case $1<p \leq 2$ this follows immediately combining Lemmas 4.2 and 4.1. In the case $p \geq 2$ we can apply Lemma 4.2 for $\tilde{p}=2$ to the function $v$. Assuming $x_{0}=0$ and $\rho=1$ (scaling argument, $B:=B_{1}(0)$ ) we get then an $(\mathcal{A}, m)$-harmonic $h \in W^{m, 2}\left(B, \mathbb{R}^{N}\right)$ with

$$
f_{B}\left|D^{m} h\right|^{2} \mathrm{~d} x \leq 1 \quad \text { and } \quad f_{B} \sum_{k=0}^{m-1}\left|D^{k}(v-\gamma h)\right|^{2} \leq \tilde{\varepsilon} \gamma^{2},
$$

$\tilde{\varepsilon}$ will be chosen later.

Without loss of generality we may assume $f_{B_{1 / 2}} D^{k}(v-\gamma h)=0$ for $k=0, \ldots, m-1$. Due to Lemma 4.1 we know that $h \in C^{\infty}\left(B, \mathbb{R}^{N}\right)$ and

$$
\sup _{B_{1 / 2}}\left|D^{m} h\right|+\sup _{B_{1 / 2}}\left|D^{m+1} h\right| \leq c_{a} f_{B}\left|D^{m} h\right| \mathrm{d} x \leq c_{a} .
$$

For $p^{*}=\left\{\begin{array}{ll}\frac{n p}{n-p} & \text { if } 1 \leq p<n \\ \left.p^{*} \in\right] p, \infty[\text { fixed } & \text { if } p \geq n\end{array}\right.$ we define $t \in\left[0,1\left[\right.\right.$ by $\frac{1}{p}=(1-t) \frac{1}{2}+t \frac{1}{p^{*}}$. Applying the SobolevPoincaré inequality we conclude for $k=0, \ldots, m-1$

$$
\begin{aligned}
f_{B_{1 / 2}}\left|D^{k}(v-\gamma h)\right|^{p} \mathrm{~d} x & \leq\left(f_{B_{1 / 2}}\left|D^{k}(v-\gamma h)\right|^{2} \mathrm{~d} x\right)^{(1-t) \frac{p}{2}}\left(f_{B_{1 / 2}}\left|D^{k}(v-\gamma h)\right|^{p^{*}} \mathrm{~d} x\right)^{\frac{t p}{p^{*}}} \\
& \leq c\left(\gamma^{2} \tilde{\varepsilon}\right)^{(1-t) \frac{p}{2}}\left(f_{B_{1 / 2}}\left|D^{k+1}(v-\gamma h)\right|^{p} \mathrm{~d} x\right)^{t} \\
& \leq c_{k}\left(\gamma^{2} \tilde{\varepsilon}\right)^{(1-t) \frac{p}{2}}\left(f_{B_{1 / 2}}\left(\left|D^{m} v\right|^{p}+\gamma^{p}\left|D^{m} h\right|^{p}\right) \mathrm{d} x\right)^{t} \\
& \leq c_{k}\left(\gamma^{2} \tilde{\varepsilon}\right)^{(1-t) \frac{p}{2}}\left(\gamma^{2}+\gamma^{p}\right)^{t} \leq c_{k} \gamma^{2} \tilde{\varepsilon}^{(1-t) \frac{p}{2}}
\end{aligned}
$$

Choosing $\tilde{\varepsilon}$ such that $2^{p} \sum_{k=0}^{m-1} c_{k} \tilde{\varepsilon}^{(1-t) \frac{p}{2}}+2^{p} \tilde{\varepsilon}=\varepsilon$ we get the claim. 


\section{Approximate $\mathcal{A}$-harmonicity}

If we want to apply the $(\mathcal{A}, m)$-harmonic approximation lemma of the last section to minimizers of the functional $F$ the following lemma is required. Before stating this lemma we define the excess functional for the function $u \in W^{m, p}\left(B_{\rho}\left(x_{0}\right), \mathbb{R}^{N}\right)$ as

$$
\Phi\left(x_{0}, \rho, D^{m} P\right)=\left(f_{B_{\rho}\left(x_{0}\right)}\left|V_{p}\left(D^{m} u\right)-V_{p}\left(D^{m} P\right)\right|^{2} \mathrm{~d} x\right)^{\frac{1}{2}},
$$

where $P$ is a polynomial of degree $\leq m$ and $B_{\rho}\left(x_{0}\right) \subset \subset \Omega$.

Lemma 5.1 (approximate $(\mathcal{A}, m)$-harmonicity). Let $u \in W^{m, p}\left(\Omega, \mathbb{R}^{N}\right)$ be a $W^{m, p}$-minimizer of the functional $F[u]=\int_{\Omega} f\left(D^{m} u\right) \mathrm{d} x$ where $f$ satisfies the assumptions $(\mathrm{H} 1)$ and $(\mathrm{H} 3 \mathrm{~s})$ with $q \leq p+1$. Then there exists for every $M>0$ a constant $c_{e}=c_{e}\left(n, N, p, q, L, M, K_{M+1}\right)$ such that for every ball $B_{\rho}\left(x_{0}\right) \subset \subset \Omega$ and for every polynomial $P$ of degree $\leq m$ with $\left|D^{m} P\right| \leq M$ we have

$$
\left|f_{B_{\rho}\left(x_{0}\right)} D^{2} f\left(D^{m} P\right)\left(D^{m}(u-P), D^{m} \varphi\right) \mathrm{d} x\right| \leq c_{e}\left(\Phi \sqrt{\nu_{M}(\Phi)}+\Phi^{2}\right) \sup _{B_{\rho}\left(x_{0}\right)}\left|D^{m} \varphi\right|
$$

for all $\varphi \in C_{0}^{\infty}\left(B_{\rho}\left(x_{0}\right), \mathbb{R}^{N}\right)$. Here we have abbreviated $\Phi\left(x_{0}, \rho, D^{m} P\right)$ by $\Phi$ and $\nu_{M}$ denotes the modulus of continuity from Remark 1.1 (4).

Proof. Since $q \leq p+1$ holds and since $f$ satisfies (1.2), the following Euler equation holds for all $\varphi \in C_{0}^{\infty}\left(\Omega, \mathbb{R}^{N}\right)$ :

$$
\int_{\Omega} D f\left(D^{m} u\right) D^{m} \varphi \mathrm{d} x=0
$$

Setting $v=u-P$ we can derive from (5.3)

$$
\begin{aligned}
\left|\int_{B_{\rho}\left(x_{0}\right)} D^{2} f\left(D^{m} P\right)\left(D^{m} v, D^{m} \varphi\right) \mathrm{d} x\right| & \leq \int_{B_{\rho}\left(x_{0}\right)}\left|\int_{0}^{1}\left(D^{2} f\left(D^{m} P\right)-D^{2} f\left(D^{m} P+t D^{m} v\right)\right) \mathrm{d} t\left(D^{m} v, D^{m} \varphi\right)\right| \mathrm{d} x \\
& =\underset{B_{\rho}\left(x_{0}\right)}{f} I \mathrm{~d} x
\end{aligned}
$$

To estimate $I$ we distinguish the cases $\left|D^{m} v\right| \leq 1$ and $\left|D^{m} v\right|>1$ on the ball $B_{\rho}\left(x_{0}\right)$.

Case 1: $x \in B_{\rho}\left(x_{0}\right) \cap\left\{\left|D^{m} v\right| \leq 1\right\}$. Since $\left|D^{m} P+t D^{m} v\right| \leq M+1$ we can use that $D^{2} f(B)$ is bounded on $\left\{B \in \bigodot^{m}\left(\mathbb{R}^{n}, \mathbb{R}^{N}\right):|B| \leq M+1\right\}$. We obtain with (1.4), (1.5), Lemma 2.1 and the fact that $\nu_{M}$ is a monotone non-decreasing function

$$
\begin{aligned}
I & \leq \sup _{B_{\rho}\left(x_{0}\right)}\left|D^{m} \varphi\right| \sqrt{2 K_{M+1}} \int_{0}^{1} \sqrt{\nu_{M}\left(t\left|D^{m} v\right|\right)}\left|D^{m} v\right| \mathrm{d} t \\
& \leq c \sup _{B_{\rho}\left(x_{0}\right)}\left|D^{m} \varphi\right| \sqrt{\nu_{M}\left(\left|V_{p}\left(D^{m} u\right)-V_{p}\left(D^{m} P\right)\right|\right)}\left|V_{p}\left(D^{m} u\right)-V_{p}\left(D^{m} P\right)\right|
\end{aligned}
$$

where $c=c\left(n, N, p, M, K_{M+1}\right)$. 
Case 2: $x \in B_{\rho}\left(x_{0}\right) \cap\left\{\left|D^{m} v\right|>1\right\}$. Using the fact $\left|D^{2} f\left(D^{m} P\right)\right| \leq K_{M}$ and inequality (1.2) (recall that $q \leq p+1)$ we see that

$$
\begin{aligned}
I & =\left|D^{2} f\left(D^{m} P\right)\left(D^{m} v, D^{m} \varphi\right)+D f\left(D^{m} P\right) D^{m} \varphi-D f\left(D^{m} u\right) D^{m} \varphi\right| \\
& \leq \sup _{B_{\rho}\left(x_{0}\right)}\left|D^{m} \varphi\right|\left(K_{M}\left|D^{m} v\right|+c\left|D^{m} v\right|^{q-1}\right) \leq c \sup _{B_{\rho}\left(x_{0}\right)}\left|D^{m} \varphi\right|\left|D^{m} v\right|^{p} \\
& \leq c \sup _{B_{\rho}\left(x_{0}\right)}\left|D^{m} \varphi\right|\left|V_{p}\left(D^{m} u\right)-V_{p}\left(D^{m} P\right)\right|^{2},
\end{aligned}
$$

where the constant $c$ depends on $n, N, p, q, L, M$ and $K_{M}$.

Returning to the general case we apply the inequalities of Hölder and Jensen to conclude $\left(\nu_{M}\right.$ concave and non-decreasing)

$$
\left|f_{B_{\rho}\left(x_{0}\right)} D^{2} f\left(D^{m} P\right)\left(D^{m}(u-P), D^{m} \varphi\right) \mathrm{d} x\right| \leq c_{e}\left(\Phi \sqrt{\nu_{M}(\Phi)}+\Phi^{2}\right) \sup _{B_{\rho}\left(x_{0}\right)}\left|D^{m} \varphi\right| .
$$

\section{Proof of Theorem 1.2}

In this section we prove the main theorem. For $B_{\rho}\left(x_{0}\right) \subset \subset \Omega$ we set

$$
\Phi(\rho):=\Phi\left(x_{0}, \rho,\left(D^{m} u\right)_{x_{0}, \rho}\right)=\left(f_{B_{\rho}\left(x_{0}\right)}\left|V_{p}\left(D^{m} u\right)-V_{p}\left(\left(D^{m} u\right)_{x_{0}, \rho}\right)\right|^{2} \mathrm{~d} x\right)^{\frac{1}{2}} .
$$

We next derive an excess-improvement estimate assuming that the excess $\Phi(\rho)$ is initially sufficient small.

Lemma 6.1 (excess-improvement). Let $M>0$ be fixed. For every $\left.\vartheta \in] 0, \frac{1}{4}\right]$ there exists $\delta=\delta\left(n, N, m, \lambda_{M}\right.$, $\left.K_{M}, \vartheta, p\right)$ such that if the following smallness conditions are satisfied

(i) $\left|\left(D^{m} u\right)_{x_{0}, \rho}\right| \leq M ; \quad\left|\left(D^{m} u\right)_{x_{0}, \vartheta \rho}\right| \leq M$;

(ii) $\sqrt{\nu_{M}(\Phi(\rho))}+\Phi(\rho) \leq \delta / 2$;

(iii) $c_{1} c_{e} c_{a} \Phi(\rho) \leq 1$;

(iv) $\vartheta^{-\frac{n^{2}}{n-1}} \Phi^{2 \frac{1}{n-1}}(\rho) \leq \vartheta^{2}$

then the following excess improvement holds:

$$
\Phi^{2}\left(x_{0}, \vartheta \rho,\left(D^{m} u\right)_{x_{0}, \vartheta \rho}\right) \leq c_{\operatorname{dec}} \vartheta^{2} \Phi^{2}\left(x_{0}, \rho,\left(D^{m} u\right)_{x_{0}, \rho}\right),
$$

where $c_{\mathrm{dec}}=c_{\mathrm{dec}}\left(n, N, m, p, q, L, \lambda_{M}, M, K_{M+1}\right)$. The constants $c_{1}, c_{a}$ and $c_{e}$ denote the constants from Lemma 2.1 (iv), Corollary 4.3 and Lemma 5.1.

Proof. We define

$$
w=u-\frac{1}{m !}\left(D^{m} u\right)_{x_{0}, \rho}\left(x-x_{0}\right)^{m} \quad \text { and } \quad \gamma=c_{1} c_{e} \Phi(\rho),
$$

where we have $\gamma \leq 1$ from the smallness condition (iii). Now we want to show that the conditions of Corollary 4.3 are fulfilled for $\mathcal{A}=D^{2} f\left(\left(D^{m} u\right)_{x_{0}, \rho}\right)$ and $v=w$. We know from (1.3) and (1.4) that $D^{2} f\left(\left(D^{m} u\right)_{x_{0}, \rho}\right)$ is elliptic in the sense of Legendre-Hadamard with ellipticity constant $\frac{2 \lambda_{M}}{m !}$ and upper bound $K=K_{M}=\sup _{|A| \leq M}\left|D^{2} f(A)\right|$. For $\left.\vartheta \in] 0, \frac{1}{4}\right]$ we set $\varepsilon=\vartheta^{n+2+\max \{2, p\}}$ and denote with $\delta=\delta\left(n, N, m, \lambda_{M}, K_{M}, \varepsilon\right)=\delta\left(n, N, m, \lambda_{M}, K_{M}, \vartheta, p\right)$ the constant from Corollary 4.3. Lemma 2.1 (vi) (recall $\left.\left|\left(D^{m} u\right)_{x_{0}, \rho}\right| \leq M\right)$ and $\gamma=c_{1} c_{e} \Phi(\rho)$ imply:

$$
\underset{B_{\rho}\left(x_{0}\right)}{f}\left|V_{p}\left(D^{m} w\right)\right|^{2} \mathrm{~d} x \leq c_{1}(p, M) \underset{B_{\rho}\left(x_{0}\right)}{f}\left|V_{p}\left(D^{m} u\right)-V_{p}\left(\left(D^{m} u\right)_{x_{0}, \rho}\right)\right|^{2} \mathrm{~d} x \leq \gamma^{2} .
$$


Furthermore, from Lemma 5.1 with $\mathcal{A}=D^{2} f\left(\left(D^{m} u\right)_{x_{0}, \rho}\right)$, the definition $\gamma=c_{1} c_{e} \Phi(\rho)$, and the smallness condition $\sqrt{\nu_{M}(\Phi(\rho))}+\Phi(\rho) \leq \delta / 2$ we infer:

$$
\left|f_{B_{\rho}\left(x_{0}\right)} D^{2} f\left(\left(D^{m} u\right)_{x_{0}, \rho}\right)\left(D^{m} w, D^{m} \varphi\right) \mathrm{d} x\right| \leq c_{e}\left(\sqrt{\nu_{M}(\Phi(\rho))} \Phi(\rho)+\Phi^{2}(\rho)\right) \sup _{B_{\rho}\left(x_{0}\right)}\left|D^{m} \varphi\right| \leq \gamma \delta \sup _{B_{\rho}\left(x_{0}\right)}\left|D^{m} \varphi\right| .
$$

Hence the assumptions of Corollary 4.3 are fulfilled and it follows that there exists a $D^{2} f\left(\left(D^{m} u\right)_{x_{0}, \rho}\right)$-harmonic function $h \in C^{\infty}\left(B_{\rho}\left(x_{0}\right), \mathbb{R}^{N}\right)$ such that

$$
\sup _{B_{\rho / 2}\left(x_{0}\right)}\left|D^{m} h\right|+\rho \sup _{B_{\rho / 2}\left(x_{0}\right)}\left|D^{m+1} h\right| \leq c_{a} \quad \text { and }{\underset{B}{B_{\rho / 2}\left(x_{0}\right)}}_{f} \sum_{k=0}^{m-1}\left|V_{p}\left(\frac{D^{k}(w-\gamma h)}{\rho}\right)\right|^{2} \mathrm{~d} x \leq \gamma^{2} \varepsilon .
$$

With Taylor's theorem applied to $D^{m-1} h$ on $B_{2 \vartheta \rho}$ we obtain the estimate

$$
\sup _{B_{2 \vartheta \rho}\left(x_{0}\right)}\left|D^{m-1} h(x)-D^{m-1} h\left(x_{0}\right)-D^{m} h\left(x_{0}\right)\left(x-x_{0}\right)\right| \leq \frac{1}{2}(2 \vartheta \rho)^{2} \sup _{B_{\rho / 2}\left(x_{0}\right)}\left|D^{m+1} h\right| \leq c \vartheta^{2} \rho .
$$

Next we define $P$ as the unique polynomial of degree $m$ satisfying

$$
\begin{aligned}
f_{B_{2 \vartheta \rho}\left(x_{0}\right)} D^{k}(u-P) \mathrm{d} x & =0 \quad \text { for } k=0, \ldots, m-2 \text { and } \\
D^{m-1} P & =\gamma D^{m-1} h\left(x_{0}\right)+\left(\left(D^{m} u\right)_{x_{0}, \rho}+\gamma D^{m} h\left(x_{0}\right)\right)\left(x-x_{0}\right)
\end{aligned}
$$

and set $v=u-P$. Using Lemma 2.1 (ii) and (iii) together with the Poincaré-type inequality (2.3) and estimate (6.2) we get:

$$
\begin{aligned}
I: & \sum_{k=0}^{m-1} \underset{B_{2 \vartheta \rho}\left(x_{0}\right)}{f}\left|V_{p}\left(\frac{D^{k} v}{(2 \vartheta \rho)^{(m-k)}}\right)\right|^{2} \mathrm{~d} x \leq c \underset{B_{2 \vartheta \rho}\left(x_{0}\right)}{f}\left|V_{p}\left(\frac{D^{m-1}(u-P)}{2 \vartheta \rho}\right)\right|^{2} \mathrm{~d} x \\
\leq & c\left[\vartheta^{-n-\max \{2, p\}} \underset{B_{\rho / 2}\left(x_{0}\right)}{f}\left|V_{p}\left(\frac{D^{m-1}(w-\gamma h)}{\rho}\right)\right|^{2} \mathrm{~d} x\right. \\
& \left.+\underset{B_{2 \vartheta \rho}\left(x_{0}\right)}{f}\left|V_{p}\left(\gamma \frac{D^{m-1} h(x)-D^{m-1} h\left(x_{0}\right)-D^{m} h\left(x_{0}\right)\left(x-x_{0}\right)}{2 \vartheta \rho}\right)\right|^{2} \mathrm{~d} x\right] \\
\leq & c\left[\vartheta^{-n-\max \{2, p\}} \gamma^{2} \varepsilon+\left|V_{p}(\vartheta \gamma)\right|^{2}\right] \leq c\left[\vartheta^{-n-\max \{2, p\}} \gamma^{2} \varepsilon+(\vartheta \gamma)^{2}\right] .
\end{aligned}
$$

Moreover, Lemma 2.1 (iv) $\left(\left|\left(D^{m} u\right)_{x_{0}, \rho}\right| \leq M\right)$ and (6.2) gives us

$$
I I:=\int_{B_{2 \vartheta \rho}\left(x_{0}\right)}\left|V_{p}\left(D^{m}(u-P)\right)\right|^{2} \leq c\left(\vartheta^{-n} \Phi^{2}(\rho)+\left|V_{p}\left(\gamma D^{m} h\left(x_{0}\right)\right)\right|^{2}\right) \leq c\left(\vartheta^{-n} \Phi^{2}(\rho)+\gamma^{2}\right) .
$$

Recalling the choice $\varepsilon=\vartheta^{n+2+\max \{2, p\}}$ and the definition of $\gamma$ we finally derive

$$
I \leq c \vartheta^{2} \Phi^{2}(\rho) \quad \text { and } \quad I I \leq c \vartheta^{-n} \Phi^{2}(\rho),
$$


where $c=c\left(n, N, m, p, \lambda_{M}, K_{M}, M\right)$. With the help of Lemma 2.1 (ii) and (iv) $\left(\left|\left(D^{m} u\right)_{x_{0}, \vartheta \rho}\right| \leq M\right)$, inequality (2.2), the convexity of $\left|W_{p}\right|^{2}$ and Jensen's inequality we deduce:

$$
\begin{aligned}
\Phi^{2}(\vartheta \rho)= & \underset{B_{\vartheta \rho}\left(x_{0}\right)}{f}\left|V_{p}\left(D^{m} u\right)-V_{p}\left(\left(D^{m} u\right)_{x_{0}, \vartheta \rho}\right)\right|^{2} \mathrm{~d} x \\
\leq & c(p, n, N, M) \underset{B_{\vartheta \rho}\left(x_{0}\right)}{f}\left|V_{p}\left(D^{m} u-\left(D^{m} u\right)_{x_{0}, \vartheta \rho}\right)\right|^{2} \mathrm{~d} x \\
\leq & c \underset{B_{\vartheta \rho}\left(x_{0}\right)}{f}\left|V_{p}\left(D^{m} u-\left(D^{m} u\right)_{x_{0}, \rho}-\gamma D^{m} h\left(x_{0}\right)\right)\right|^{2} \mathrm{~d} x \\
& +c\left|W_{p}\left(\left(D^{m} u\right)_{x_{0}, \vartheta \rho}-\left(D^{m} u\right)_{x_{0}, \rho}-\gamma D^{m} h\left(x_{0}\right)\right)\right|^{2} \\
\leq & c \underset{B_{\vartheta \rho}\left(x_{0}\right)}{f}\left|V_{p}\left(D^{m} u-\left(D^{m} u\right)_{x_{0}, \rho}-\gamma D^{m} h\left(x_{0}\right)\right)\right|^{2} \mathrm{~d} x=\underset{B_{\vartheta \rho}\left(x_{0}\right)}{f}\left|V_{p}\left(D^{m} v\right)\right|^{2} \mathrm{~d} x,
\end{aligned}
$$

where $c$ depends on $n, N, M$ and $p$. Using $\left|\left(D^{m} u\right)_{x_{0}, \rho}\right| \leq M,(6.2)$, the definition of $\gamma$ and the smallness assumption (iii) we conclude

$$
\left|D^{m} P\right| \leq\left|\left(D^{m} u\right)_{x_{0}, \rho}\right|+\left|\gamma D^{m} h\left(x_{0}\right)\right| \leq M+\gamma c_{a} \leq M+c_{1} c_{e} c_{a} \Phi(\rho) \leq M+1 .
$$

Now we apply the Caccioppoli inequality (Lem. 3.1) on $B_{\vartheta \rho}\left(x_{0}\right)$ and obtain with Lemma 2.1 (ii) and the estimates for $I$ and $I I$ :

$$
\begin{aligned}
\Phi^{2}(\vartheta \rho) & \leq c \underset{B_{\vartheta \rho}\left(x_{0}\right)}{f}\left|V_{p}\left(D^{m} v\right)\right|^{2} \mathrm{~d} x \\
& \leq c\left[f_{B_{2 \vartheta \rho}\left(x_{0}\right)} \sum_{k=0}^{m-1}\left|V_{p}\left(\frac{D^{k} v}{(2 \vartheta \rho)^{m-k}}\right)\right|^{2} \mathrm{~d} x+\left(\underset{B_{2 \vartheta \rho}\left(x_{0}\right)}{f} \sum_{k=0}^{m}\left|V_{p}\left(\frac{D^{k} v}{(2 \vartheta \rho)^{m-k}}\right)\right|^{2} \mathrm{~d} x\right)^{\frac{n}{n-1}}\right] \\
& \leq c\left[I+I^{\frac{n}{n-1}}+I I^{\frac{n}{n-1}}\right] \leq c\left[\vartheta^{2} \Phi^{2}(\rho)+\vartheta^{2 \frac{n}{n-1}} \Phi^{2 \frac{n}{n-1}}(\rho)+\vartheta^{-\frac{n^{2}}{n-1}} \Phi^{2 \frac{n}{n-1}}(\rho)\right] .
\end{aligned}
$$

The smallness assumption (iv) and $\Phi(\rho) \leq 1$ imply

$$
\Phi^{2}\left(x_{0}, \vartheta \rho,\left(D^{m} u\right)_{x_{0}, \vartheta \rho}\right)=\Phi^{2}(\vartheta \rho) \leq c_{\mathrm{dec}} \vartheta^{2} \Phi^{2}(\rho)=c_{\mathrm{dec}} \vartheta^{2} \Phi^{2}\left(x_{0}, \rho,\left(D^{m} u\right)_{x_{0}, \rho}\right),
$$

where $c_{\text {dec }}$ depends on $n, N, m, p, q, L, \lambda_{M}, M$ and $K_{M+1}$.

The smallness conditions of Lemma 6.1 are fulfilled in all points $x_{0} \in \Omega_{0}$. We can now iterate inequality (6.1) of Lemma 6.1 to derive the following excess-decay estimate for $\Phi(\rho)$ (for further details see $[11,20]$ )

$$
\Phi\left(x_{0}, r,\left(D^{m} u\right)_{x_{0}, r}\right) \leq\left(\frac{r}{\rho}\right)^{\alpha} \Phi\left(x_{0}, \rho,\left(D^{m} u\right)_{x_{0}, \rho}\right)
$$

for all $0<r \leq \rho$ and $0<\alpha<1$. Using Camapanato's integral characterization of Hölder continuous functions (see e.g. [24]) and the property Lemma 2.1 (iii) of the function $V_{p}$ we complete the proof of Theorem 1.2. 


\section{REFERENCES}

[1] E. Acerbi and N. Fusco, Semicontinuity problems in the calculus of variations. Arch. Ration. Mech. Anal. 86 (1984) 125-145.

[2] E. Acerbi and N. Fusco, A regularity theorem for minimizers of quasiconvex integrals. Arch. Ration. Mech. Anal. 99 (1987) 261-281.

[3] E. Acerbi and N. Fusco, Regularity for minimizers of non-quadratic functionals: the case $1<p<2$. J. Math. Anal. Appl. 140 (1989) 115-135.

[4] E. Acerbi and N. Fusco, Partial regularity under anisotropic $(p, q)$ growth conditions. J. Differ. Equ. 107 (1994) 46-67.

[5] E. Acerbi and G. Mingione, Regularity results for a class of quasiconvex functionals with nonstandard growth. Ann. Scuola Norm. Sup. Pisa Cl. Sci. IV 30 (2001) 311-339.

[6] J.M. Ball and F. Murat, $W^{1, p}$-quasiconvexity and variational problems for multiple integrals. J. Funct. Anal. 58 (1984) $225-253$.

[7] M. Bildhauer and M. Fuchs, Partial regularity for variational integrals with $(s, \mu, q)$-growth. Calc. Var. Partial Differ. Equ. 13 (2001) 537-560.

[8] M. Bildhauer and M. Fuchs, $C^{1, \alpha}$-solutions to non-autonomous anisotropic variational problems. Calc. Var. Partial Differ. Equ. 24 (2005) 309-340.

[9] G. Bouchitté, I. Fonseca and J. Malý, The effective bulk energy of the relaxed energy of multiple integrals below the growth exponent. Proc. Roy. Soc. Edinburgh Sect. A 128 (1998) 463-479.

[10] M. Carozza and A. Passarelli di Napoli, Partial regularity for anisotropic functionals of higher order. ESAIM: COCV 13 (2007) 692-706.

[11] M. Carozza, N. Fusco and G. Mingione, Partial regularity of minimizers of quasiconvex integrals with subquadratic growth. Ann. Mat. Pura Appl. IV 175 (1998) 141-164.

[12] G. Cupini, M. Guidorzi and E. Mascolo, Regularity of minimizers of vectorial integrals with $p-q$ growth. Nonlinear Anal., Theory Methods Appl. 54 (2003) 591-616.

[13] F. Duzaar and M. Kronz, Regularity of $\omega$-minimizers of quasi-convex variational integrals with polynomial growth. Differ. Geom. Appl. 17 (2002) 139-152.

[14] F. Duzaar and K. Steffen, Optimal interior and boundary regularity for almost minimizers to elliptic variational integrals. J. Reine Angew. Math. 546 (2002) 73-138.

[15] F. Duzaar, A. Gastel and J. Grotowski, Partial regularity for almost minimizers of quasi-convex integrals. SIAM J. Math. Anal. 32 (2000) 665-687.

[16] F. Duzaar, J. Grotowski and M. Kronz, Regularity of almost minimizers of quasi-convex variational integrals with subquadratic growth. Ann. Mat. Pura Appl. IV 184 (2005) 421-448.

[17] L. Esposito and G. Mingione, Relaxation results for higher order integrals below the natural growth exponent. Differ. Integral Equ. 15 (2002) 671-696.

[18] L. Esposito, F. Leonetti and G. Mingione, Regularity results for minimizers of irregular integrals with $(p, q)$ growth. Forum Math. 14 (2002) 245-272.

[19] L. Esposito, F. Leonetti and G. Mingione, Sharp regularity for functionals with (p,q) growth. J. Differ. Equ. 204 (2004) 5-55.

[20] L.C. Evans, Quasiconvexity and partial regularity in the calculus of variations. Arch. Ration. Mech. Anal. 95 (1986) $227-252$.

[21] I. Fonseca and J. Malý, Relaxation of multiple integrals below the growth exponent. Ann. Inst. Henri Poincaré Anal. Non Linéaire 14 (1997) 309-338.

[22] I. Fonseca and J. Malý, From jacobian to hessian: distributional form and relaxation. Riv. Mat. Univ. Parma 4 (2005) 45-74.

[23] N. Fusco and J. Hutchinson, $C^{1, \alpha}$ partial regularity of functions minimising quasiconvex integrals. Manuscr. Math. 54 (1984) $121-143$.

[24] M. Giaquinta, Multiple integrals in the calculus of variations and nonlinear elliptic systems. Princeton University Press, Princeton (1983).

[25] M. Giaquinta, Growth conditions and regularity, a counterexample. Manuscr. Math. 59 (1987) 245-248.

[26] M. Giaquinta and G. Modica, Partial regularity of minimizers of quasiconvex integrals. Ann. Inst. Henri Poincaré Anal. Non Linéaire 3 (1986) 185-208.

[27] M. Guidorzi, A remark on partial regularity of minimizers of quasiconvex integrals of higher order. Rend. Ist. Mat. Univ. Trieste 32 (2000) 1-24.

[28] M. Guidorzi and L. Poggiolini, Lower semicontinuity of quasiconvex integrals of higher order. NoDEA 6 (1999) 227-246.

[29] M.C. Hong, Some remarks on the minimizers of variational integrals with non standard growth conditions. Boll. Un. Mat. Ital. A 6 (1992) 91-101.

[30] J. Kristensen, Lower semicontinuity in Sobolev spaces below the growth exponent of the integrand. Proc. Roy. Soc. Edinburgh Sect. A 127 (1997) 797-817.

[31] J. Kristensen and G. Mingione, The singular set of lipschitzian minima of multiple integrals. Arch. Ration. Mech. Anal. 184 (2007) 341-369.

[32] M. Kronz, Partial regularity results for minimizers of quasiconvex functionals of higher order. Ann. Inst. Henri Poincaré Anal. Non Linéaire 19 (2002) 81-112. 
[33] P. Marcellini, Approximation of quasiconvex functions and lower semicontinuity of multiple integrals. Manuscr. Math. 51 (1985) 1-28.

[34] P. Marcellini, On the definition and the lower semicontinuity of certain quasiconvex integrals. Ann. Inst. Henri Poincaré Anal. Non Linéaire 3 (1986) 391-409.

[35] P. Marcellini, Un exemple de solution discontinue d'un problème variationnel dans le cas scalaire. Preprint Istituto Matematico U. Dini, Universita' di Firenze (1987/1988), n. 11.

[36] P. Marcellini, Regularity of minimizers of integrals of the calculus of variations with non-standard growth conditions. Arch. Ration. Mech. Anal. 105 (1989) 267-284.

[37] P. Marcellini, Regularity and existence of solutions of elliptic equations with $p, q$-growth conditions. J. Differ. Equ. 90 (1991) $1-30$.

[38] P. Marcellini, Everywhere regularity for a class of elliptic systems without growth conditions. Ann. Scuola Norm. Sup. Pisa Cl. Sci. IV $2 \mathbf{3}$ (1996) 1-25.

[39] N.G. Meyers, Quasi-convexity and lower semi-continuity of multiple variational integrals of any order. Trans. Am. Math. Soc. 119 (1965) 125-149.

[40] C.B. Morrey, Quasi-convexity and the lower semicontinuity of multiple integrals. Pac. J. Math. 2 (1952) $25-53$.

[41] A. Passarelli di Napoli and F. Siepe, A regularity result for a class of anisotropic systems. Rend. Ist. Mat. Univ. Trieste 28 (1996) 13-31.

[42] S. Schemm and T. Schmidt, Partial regularity of strong local minimizers of quasiconvex integrals with ( $p, q)$-growth. Proc. Roy. Soc. Edinburgh Sect. A 139 (2009) 595-621.

[43] T. Schmidt, Regularity of minimizers of $W^{1, p}$-quasiconvex variational integrals with $(p, q)$-growth. Calc. Var. Partial Differ. Equ. 32 (2008) 1-24.

[44] T. Schmidt, Regularity of relaxed minimizers of quasiconvex variational integrals with (p, q)-growth. Arch. Ration. Mech. Anal. 193 (2009) 311-337.

[45] F. Siepe and M. Guidorzi, Partial regularity for quasiconvex integrals of any order. Ric. Mat. 52 (2003) 31-54. 